\title{
Cojumps in Stock Prices: Empirical Evidence
}

\author{
Dudley Gilder ${ }^{\mathrm{a}, *}$, Mark B. Shackleton ${ }^{\mathrm{b}}$, Stephen J. Taylor ${ }^{\mathrm{b}}$ \\ ${ }^{a}$ Finance and Accounting Group, Aston Business School, Aston University, Birmingham, \\ B4 $7 E T$ \\ ${ }^{b}$ Department of Accounting and Finance, Lancaster University Management School, \\ Lancaster University, Lancaster, LA1 $4 Y X, U K$
}

\begin{abstract}
We examine contemporaneous jumps (cojumps) among individual stocks and a proxy for the market portfolio. We show, through a Monte Carlo study, that using intraday jump tests and a coexceedance criterion to detect cojumps has a power similar to the cojump test proposed by Bollerslev et al. (2008). However, we also show that we should not expect to detect all common jumps comprising a cojump when using such coexceedance based detection methods. Empirically, we provide evidence of an association between jumps in the market portfolio and cojumps in the underlying stocks. Consistent with our Monte Carlo evidence, moderate numbers of stocks are often detected to be involved in these (systematic) cojumps. Importantly, the results suggest that market-level news is able to generate simultaneous large jumps in individual stocks. We also find evidence of an association between systematic cojumps and Federal Funds Target Rate announcements.

Keywords: High-Frequency Stock Prices, Non-parametric Jump Tests,

Realised Volatility, Macroeconomic News

JEL: C12, C58, G10
\end{abstract}

\section{Introduction}

Recently, non-parametric tests employing high-frequency data have been developed to detect whether, in addition to a continuous diffusion, asset prices are driven by discontinuous jumps. The seminal work in this area is Barndorff-

\footnotetext{
${ }^{*}$ Corresponding author. E mail: d.gilder@aston.ac.uk
} 
Nielsen and Shephard (2004a, 2006) (BNS), who developed a technique to identify jumps which relies on separating realised measures of volatility into a component driven by continuous price changes and another component driven by jumps. Subsequently, many additional tests based on non-parametric volatility estimators have been developed (Jiang and Oomen (2008), Andersen et al. (2010b), Corsi et al. (2010), Podolskij and Ziggel (2010), Christensen et al. (2011)). All of these non-parametric tests identify whether jumps are present over a given interval of time, which is usually selected to be one day. Tests which explicitly identify intraday jumps have been developed by Andersen et al. (2007) (ABD), Lee and Mykland (2008) (LM) and Andersen et al. (2010a). Application of these non-parametric tests to various markets has supported the presence of price jumps. For example, jumps in equity indices and individual stocks have been documented in Huang and Tauchen (2005), Andersen et al. (2007), Lee and Mykland (2008), Lee and Hannig (2010), Lahaye et al. (2011) and Evans (2011), among others. Evidence for the presence of jumps in foreign exchange and Treasury bond markets is given by Dungey et al. (2009), Jiang et al. (2011) and Dungey and Hvozdyk (2012).

However, there has been little research into cojumps; that is, the tendency for jumps to arrive simultaneously. In this paper we investigate two hypotheses concerning cojumps in stock prices. The first is that there should be an association between jumps in the market portfolio and common jumps, or cojumps, among the underlying stocks (Hypothesis 1). We refer to cojumps which involve the market portfolio as systematic cojumps, since they represent non-diversifiable cojumps. Intuitively, a systematic cojump results from market-level news initiating a cojump amongst the underlying stocks, which is ultimately reflected as a jump in the market portfolio.

Clearly, because they cannot be diversified away, systematic cojumps have 
important implications for portfolio selection and hedging. For example, AïtSahalia et al. (2009) examine the consequences of common jumps (i.e., systematic cojumps) amongst a set of assets in the context of dynamic portfolio choice. They show that an investor's portfolio decisions will be dominated by a concern to maintain exposure to jump risk below a desired level ${ }^{1}$. Merton (1976) provides an early demonstration of the role systematic cojumps have in forming risk-neutral hedging portfolios of options and stocks. It is shown that, due to the non-linear relationship between option prices and the prices of their underlying asset, it is not possible to form risk-neutral hedging portfolios if systematic cojumps occur. However, hedging portfolios can be formed that are risk-neutral on average if all jumps are idiosyncratic.

Despite their importance, there has been little empirical work examining the existence of systematic cojumps. Bollerslev et al. (2008) (BLT) were the first to examine Hypothesis 1 with non-parametric jump tests. They apply the BNS test to a panel of stock prices and a proxy for the market portfolio and find only a weak association between the jumps they detect in the underlying stocks and their market portfolio proxy. They highlight that non-parametric jump tests are only able to detect large jumps. Here, "large jump" is a relative term. A jump is large, and therefore detectable, if it is large relative to the volatility of the continuous diffusion component of stock prices. BLT argue that jumps are masked by additional volatility introduced by idiosyncratic price changes in the individual stocks. They then proceed to develop an intraday cojump test that is able to detect small (co)jumps in large panels of stock prices.

\footnotetext{
${ }^{1}$ Das and Uppal (2004) examine the effect of systemic jumps (common jumps across international markets) on optimal portfolios of international stocks. They find that the portfolios of investors who take systemic jumps into account should be levered to a lesser degree relative to the portfolios of investors who do not take systemic jumps into account.
} 
BLT conclude that systematic cojumps tend to be generated by small jumps in individual stocks and that firm-specific news is primarily responsible for generating large stock price jumps.

Todorov and Bollerslev (2010) develop an estimator that separates the CAPM beta into a continuous beta, driven by covariance between the continuous diffusion components of stock prices and the market portfolio, and a jump beta, driven by cojumps between the market portfolio and individual stocks. Although they provide empirical estimates of jump betas, the estimators do not require the identification of systematic cojumps directly. The identification of common large jumps in the market portfolio and individual stocks is also important in Bollerslev et al. (2013), who measure the dependence between the tails of systematic and non-systematic jump distributions. However, the issue of whether there is a true association between jumps in the market portfolio and cojumps in the individual stocks is not addressed. Their primary concern is with cojumps in bivariate series consisting of an individual stock and the market portfolio, rather than cojumps in large panels of stocks.

Our analysis is similar to BLT in that we apply univariate jump tests to a panel of stocks to examine cojumps. We refer to these as coexceedance based detection methods. However, we differ in that we concentrate on using the intraday jump tests of ABD, LM and Andersen et al. (2010a) in our coexceedance based detection methods and we compare the efficacy of these tests to the BLT test. We also focus on examining the number and timing of jumps directly, whereas BLT's evidence of a weak association between jumps in their market portfolio proxy and jumps in the individual stocks is based on the correlation between BNS test statistics.

Our first contribution is to demonstrate, through a Monte Carlo simulation study, that it is possible to detect cojumps using coexceedance based detection 
methods. However, we show that we should not expect to detect all the common jumps comprising a cojump, even if the jumps in all stocks are large. We also provide evidence that our intraday coexceedance based detection methods have a similar power to the intraday BLT test for large and small (co)jumps.

Our second contribution is to document an association between jumps in (a proxy for) the market portfolio and cojumps in the underlying stocks using our coexceedance based detection methods, i.e., we document evidence in support of Hypothesis 1. Although BLT document evidence of cojumps amongst a panel of stocks, they do not demonstrate explicitly that they are associated with jumps in their market portfolio proxy. The number of stocks detected to be involved in the systematic cojumps identified by our coexceedance based detection methods is often moderate relative to the number of stocks included in our sample. However, given the evidence from our Monte Carlo study, we argue that the moderate numbers are consistent with the occurrence of a systematic cojump; the true number of stocks involved is likely to be much greater. In addition, we demonstrate that it is very unlikely that we would detect these moderate numbers of stocks cojumping if the jump arrivals in the individual stocks were independent. Importantly, the results suggest there is an association between large jumps in the market portfolio and large jumps in the underlying stocks. In other words, market-level news is able to generate simultaneous large jumps in stock prices.

We also document non-systematic cojumps which involve relatively large numbers of stocks. We are unable to explain these as being industry-specific cojumps or related to jumps in (proxies for) the Fama and French (FF) SMB and HML factors (Fama and French, 1992, 1993). We conclude these nonsystematic cojumps are misclassified and result from a failure to detect some jumps in the market portfolio. Hence, systematic cojumps are likely to be 
more common than is suggested by the number of jumps detected in the market portfolio. This is in agreement with BLT, who make a similar conclusion based on results from their test.

Finally, we examine whether the BLT test and the coexceedance based detection methods offer substitute approaches to cojump detection. We do this by analysing the intersection between the cojumps detected by each approach. Reassuringly, there is reasonable intersection between the systematic cojumps detected by the coexceedance based detection methods and the cojumps detected by the BLT test. However, there are occasions on which the two approaches disagree. In sum, the analysis suggests the approaches could be combined in a manner similar to that recommended by Dumitru and Urga (2012) for univariate jump tests. If we would like to be more certain that they are detecting true cojumps, then we should only select cojumps detected by both the coexceedance based detection methods and the BLT test. On the other hand, if we would like to maximise the power of cojump detection, then we should select cojumps detected by either the coexceedance based detection methods or the BLT test.

The second hypothesis we examine is that systematic cojumps will be associated with the release of macroeconomic news (Hypothesis 2). Since macroeconomic news can be considered to have a market-level influence, it is an obvious candidate as a source of systematic cojumps. An association between macroeconomic news and the arrival of (co)jumps has been demonstrated in various markets. For example, Dungey et al. (2009) and Dungey and Hvozdyk (2012) document a relationship between cojumps and macroeconomic news releases in bond markets; Lahaye et al. (2011) investigate cojumps between an equity index, bond index and exchange rates and link them to macroeconomic news announcements; and Evans (2011) demonstrates an association 
between jumps in equity futures and macroeconomic news. Bajgrowicz and Scaillet (2011) do examine jumps in individual stock prices and demonstrate an association with macroeconomic news, but they rely on the daily BNS jump test and do not distinguish whether such an association exists with systematic cojumps. BLT conjecture an association exists between cojumps in stock prices and macroeconomic news based on the intraday timing at which they detect cojumps, but do not demonstrate a direct link with macroeconomic news announcements.

In contrast, we examine whether the timing of systematic cojumps in stocks are associated with the timing of macroeconomic news released during trading hours ${ }^{2}$. We find some support for Hypothesis 2. There is evidence of an association between systematic cojumps and the Federal Funds Target Rate announcement. Evidence of an association with other macroeconomic announcements released during trading hours is tentative.

The remainder of the paper is organised as follows. Section 2 describes the (co)jump detection methods. Section 3 presents results from our Monte Carlo simulation study. Section 4 describes our data. Results from applying the jump tests to the individual stocks and our market portfolio proxy are given in Section 5, whilst Section 6 presents our cojump results. The association between macroeconomic news and (co)jumps is analysed in Section 7. Section 8 concludes.

\footnotetext{
${ }^{2}$ Note, we do not examine the surprise component of macroeconomic news announcements, only their timing.
} 


\section{Jump and Cojump Identification}

\subsection{Nonparametric Volatility Metrics}

Essential to nonparametric jump tests are two high-frequency volatility metrics: realised variance $(\mathrm{RV})$ and realised bipower variation $(\mathrm{BV})^{3}$. The theoretical relevance of these volatility metrics is based on the assumption that prices follow a semi-martingale, which ensures no-arbitrage. Log prices, $p(t)$, are assumed to be generated by,

$$
\mathrm{d} p(t)=\mu(t) \mathrm{d} t+\sigma(t) \mathrm{d} W(t)+\kappa(t) \mathrm{d} N(t),
$$

where $\mu(t)$ is a process of finite variation, $\sigma(t)$ is a càdlàg stochastic volatility process, $W(t)$ is a Brownian motion, $N(t)$ is a finite activity counting process with intensity $\lambda(t)$, which may also be stochastic, and $\kappa(t)$ represents the random jump sizes.

Given a set of $M+1$ intraday log prices for day $t$, equally spaced in time, $\left\{p_{t, 0}, \ldots, p_{t, M}\right\}, M$ intraday returns can be formed by $r_{t, i}=p_{t, i}-p_{t, i-1}, i=$ $1, \ldots, M$. For day $t, \mathrm{RV}$ can then be computed from,

$$
R V_{t}=\sum_{i=1}^{M} r_{t, i}^{2}
$$

The probability limit of RV equals the quadratic variation (QV) of the process generating log prices on day $t$ (Andersen and Bollerslev, 1998a; Andersen et al., 2001, 2003). In the absence of jumps, QV is equivalent to integrated variance

\footnotetext{
${ }^{3}$ For a review of RV see McAleer and Medeiros (2008) and Andersen et al. (2009).
} 
(IV),

$$
\operatorname{plim}_{M \rightarrow \infty} R V_{t}=Q V_{t}=I V_{t}=\int_{t-1}^{t} \sigma^{2}(u) \mathrm{d} u,
$$

with day $t$ interpreted as commencing at time $t-1$ and concluding at time $t$. If jumps are present, then,

$$
\operatorname{plim}_{M \rightarrow \infty} R V_{t}=Q V_{t}=\int_{t-1}^{t} \sigma^{2}(u) \mathrm{d} u+\sum_{(t-1) \leq s \leq t}(\Delta p(s))^{2},
$$

where $\Delta p(s)$ represents the instantaneous change in the log price resulting from a jump at time $s$. Therefore, when there are jumps in prices, $R V_{t}$ incorporates both the integrated variance for day $t$ and the sum of the squared jumps realised during day $t$.

In contrast, BV is robust to jumps and is given by (Barndorff-Nielsen and Shephard, 2004a, 2006),

$$
B V_{t}=\mu_{1}^{-2}\left(\frac{M}{M-1}\right) \sum_{i=2}^{M}\left|r_{t, i}\right|\left|r_{t, i-1}\right|,
$$

where, $\mu_{p}=\mathbb{E}\left[|u|^{p}\right]$ and $u \sim \mathcal{N}(0,1)$. Hence, $\mu_{1}^{-2}=(\mathbb{E}[|u|])^{-2}=\pi / 2$. The probability limit is,

$$
\operatorname{plim}_{M \rightarrow \infty} B V_{t}=I V_{t},
$$

irrespective of whether jumps are present or not ${ }^{4}$.

\footnotetext{
${ }^{4}$ Andersen et al. (2010b) have suggested MinRV and MedRV as alternatives to BV. These are more robust in finite samples where many of the intraday returns take a value of zero. However, we did not find any benefit in adopting these alternative IV estimators, empirically or in our Monte Carlo Simulation study
} 


\subsection{Jump Tests}

We employ the daily non-parametric jump test of Barndorff-Nielsen and Shephard (2004a, 2006) and the non-parametric intraday jump tests of Andersen et al. (2007), Lee and Mykland (2008) and Andersen et al. (2010a).

We apply the ratio form of the BNS test as recommended by Huang and Tauchen (2005). At least one jump is detected for day $t$ if,

$$
Z_{B N S, t}=\frac{\frac{R V_{t}-B V_{t}}{R V_{t}}}{\sqrt{\left(\left(\frac{\pi}{2}\right)^{2}+\pi-5\right) \frac{1}{M} \max \left(1, \frac{T P V_{t}}{B V_{t}^{2}}\right)}}>\Phi_{1-\alpha}^{-1}
$$

where,

$$
T P V_{t}=M \mu_{4 / 3}^{-3}\left(\frac{M}{M-2}\right) \sum_{i=3}^{M}\left|r_{t, i}\right|^{\frac{4}{3}}\left|r_{t, i-1}\right|^{\frac{4}{3}}\left|r_{t, i-2}\right|^{\frac{4}{3}}
$$

and $\Phi_{1-\alpha}^{-1}$ represents the inverse of the standard normal cumulative distribution function evaluated at a cumulative probability of $1-\alpha$.

The sequential BNS test (s-BNS test) of Andersen et al. (2010a) detects intraday jumps by firstly detecting jump days using the BNS test and then selecting the maximum intraday return to be the intraday jump. To detect all intraday jumps, the procedure is repeated until the BNS test is insignificant. At each iteration, RV is re-calculated with the previously identified intraday jump/return set to zero. We modify the procedure by selecting the maximum standardised return, $\max \left(\left|r_{t, i}\right| / \sqrt{\hat{s}_{W S D, i}^{2} \cdot \Delta \cdot B V_{t}}\right)$, for $i=1, \ldots, M$, where $\Delta=1 / M$. Note, $\hat{s}_{W S D, i}^{2}$ corrects for the, U-shaped, intraday volatility pattern observed in equity markets (Wood et al. (1985), Harris (1986)). Specifically, $\hat{s}_{W S D, i}^{2}$ is the weighted standard deviation (WSD) estimator proposed by Boudt et al. (2011). To obtain $\hat{s}_{W S D, i}^{2}$, it is first necessary to estimate the shortest 
half scale estimator, Short $H_{i}$, of Boudt et al. (2011). First, define,

$$
\bar{r}_{t, i}=\frac{r_{t, i}}{\sqrt{\Delta \cdot B V_{t}}}
$$

Then,

$$
\begin{aligned}
\text { Short } H_{i} & =0.741 \cdot \min \left\{\bar{r}_{\left(h_{i}\right), i}-\bar{r}_{(1), i}, \ldots, \bar{r}_{\left(T_{i}\right), i}-\bar{r}_{\left(T_{i}-h_{i}+1\right), i}\right\} \\
h_{i} & =\left\lfloor T_{i} / 2\right\rfloor+1
\end{aligned}
$$

where $T_{i}$ is the total number of observations of intraday interval $i,\lfloor A\rfloor$ rounds $A$ to the lowest integer and the $\bar{r}_{(j), i}$ are the order statistics of $\bar{r}_{j, i}$. Then,

$$
\hat{s}_{\text {Short }, i}^{2}=\frac{M \cdot \text { Short }_{i}^{2}}{\sum_{i=1}^{M} \text { Short }_{i}^{2}} .
$$

The WSD estimator is then given by,

$$
W S D_{i}^{2}=1.081 \frac{\sum_{t=1}^{T} w_{t, i} \bar{r}_{t, i}^{2}}{\sum_{t=1}^{T} w_{t, i}}
$$

where $w_{t, i}=w\left(\frac{\bar{r}_{t, i}}{\hat{s}_{\text {Short }, i}}\right)$ and $w(z)=1$ if $z^{2} \leq 6.635$ and 0 otherwise. Finally,

$$
\hat{s}_{W S D, i}^{2}=\frac{M \cdot W S D_{i}^{2}}{\sum_{i=1}^{M} W S D_{i}^{2}} .
$$

We use the WSD intraday volatility corrector because it is one of the robustto-jumps estimators recommended by Boudt et al. (2011) and has been used by Lahaye et al. $(2011)^{5}$.

\footnotetext{
${ }^{5}$ Using alternative intraday volatility correctors had little effect on our results from the
} 
A jump is detected with the ABD test on day $t$ for intraday interval $i$ when,

$$
\left|r_{t, i}\right|>\Phi_{1-\beta / 2}^{-1} \cdot \sqrt{\hat{s}_{W S D, i}^{2} \cdot \Delta \cdot B V_{t}}
$$

where $(1-\beta)^{M}=1-\alpha$ and $\alpha$ represents the daily significance level.

To apply the LM test, first define $\mathcal{L}_{i}=r_{t, i} /\left(\hat{s}_{W S D, i} \hat{\sigma}_{L M, t, i}\right)$, where,

$$
\hat{\sigma}_{L M, t, i}^{2}=\frac{1}{K-2} \sum_{j=i-K+1}^{i-1}\left|r_{t, j}\right|\left|r_{t, j-1}\right|
$$

Lee and Mykland (2008) suggest $K=\sqrt{M \times 252}$. A jump is detected if,

$$
\left|\mathcal{L}_{i}\right|>\frac{\xi}{c \sqrt{2 \ln M}}+\frac{\sqrt{(2 \ln M)}}{c}-\frac{(\ln 4 \pi+\ln (\ln M))}{2 c \sqrt{2 \ln M}}
$$

where $c=\sqrt{2 / \pi}$ and $\xi=-\ln (-\ln (1-\alpha))^{6}$.

When a jump is detected by the ABD, LM or s-BNS tests, we assume its size dominates that of any diffusion component. Thus, the size of a jump detected on day $t$ for intraday interval $i$ is set equal to the value of the return for that interval.

Monte Carlo study or our empirical results.

${ }^{6}$ Note, in the original paper (Lee and Mykland (2008)), the numerator of the third term on the right-hand-side of (4) was printed as $-(\ln \pi+\ln (\ln M))$. This is an error and we use the correct form. We thank Ping-Chen Tsai for highlighting the correct equation. 


\subsection{Cojump Test}

We take two approaches to cojump detection. Firstly, we use the following coexceedance rule,

$$
\sum_{j=1}^{N} \mathbb{I}\left\{\operatorname{Jump}_{t, i, j}>0\right\} \begin{cases}\geq 2 & \text { Cojump } \\ \leq 1 & \text { No Cojump }\end{cases}
$$

where $\mathbb{I}\left\{\operatorname{Jump}_{t, i, j}>0\right\}$ is an indicator function taking the value 1 when a jump is detected in asset $j$ during intraday interval $i$ on day $t$. We use the coexceedance rule in conjunction with the ABD, LM, s-BNS and BNS tests to detect both intraday jumps and jump days. We refer to these as coexceedance based detection methods.

Secondly, we use the intraday cojump test of BLT ${ }^{7}$. This is used to detect intraday cojumps in a large panel of $N$ stocks. The test statistic is,

$$
Z_{m c p, t, i}=\frac{m c p_{t, i}-\overline{m c p_{t}}}{\hat{\sigma}_{B L T, t}}
$$

where,

$$
\begin{aligned}
m_{c p_{t, i}} & =\frac{2}{N(N-1)} \sum_{l=1}^{N-1} \sum_{m=l+1}^{N} \frac{r_{l, t, i}}{\hat{s}_{l, W S D, i}} \frac{r_{m, t, i}}{\hat{s}_{m, W S D, i}} \\
\overline{m c p_{t}} & =\frac{1}{M} \sum_{i=1}^{M} m c p_{t, i}, \\
\hat{\sigma}_{B L T, t} & =\sqrt{\frac{1}{M-1} \sum_{i=1}^{M}\left(m c p_{t, i}-\overline{m c p_{t}}\right)^{2}} .
\end{aligned}
$$

\footnotetext{
${ }^{7}$ We also tried applying the bivariate cojump test of Jacod and Todorov (2009). However, this test is only able to detect cojumps between two assets and therefore it was not clear how this test could contribute to our analysis of cojumps between 61 securities.
} 
Note, we differ from BLT in using intraday returns standardised by an intraday volatility corrector. We find this helps mitigate the effect of the U-shaped intraday volatility pattern. To find critical values, we follow the bootstrap procedure of BLT.

To clarify terminology, cojumps involving the market proxy are said to be systematic cojumps, whilst cojumps amongst the individual stocks that exclude the market proxy are said to be non-systematic cojumps.

\section{Monte Carlo Study}

We conduct a multivariate Monte Carlo simulation study to examine the efficacy of our cojump detection methods. The simulation results presented below are not intended to be comprehensive, but to reflect our empirical application of the cojump detection methods.

\subsection{Simulation Set-Up}

We generate log prices for 61 assets from a modified version of the multivariate model used in Barndorff-Nielsen et al. (2011). Our modifications insert a common intraday volatility pattern $\sigma_{u}(t)$ and cojumps driven by a counting process $N(t)$. When $\sigma_{u}(t)=1$ and $N(t)=0$, for all $t$, our model has the same notation and equations as Barndorff-Nielsen et al. (2011). Our simulated model is:

$$
\begin{aligned}
d p^{(j)}(t) & =\mu^{(j)} d t+\sigma_{u}(t)\left(d V^{(j)}(t)+d F^{(j)}(t)+\sigma^{(j)}(t) \psi(t) d N(t)\right) \\
d V^{(j)}(t) & =\rho^{(j)} \sigma^{(j)}(t) d W_{1}^{(j)}(t) \\
d F^{(j)}(t) & =\sqrt{1-\left(\rho^{(j)}\right)^{2}} \sigma^{(j)}(t) d W_{2}(t)
\end{aligned}
$$


where $W_{1}^{(j)}(t)$ and $W_{2}(t)$ are independent Brownian motions and $N(t)$ is an independent Poisson counting process with constant intensity, $\lambda$. In this model, $N(t)$ introduces cojumps between all assets $j=1, \ldots, N$ and $F^{(j)}$ reflects a common factor. The drift for asset $j$ is $\mu^{(j)}$ and the instantaneous volatility of the continuous components is $\sigma^{(j)}(t)=\exp \left(\beta_{0}^{(j)}+\beta_{1}^{(j)} \varrho^{(j)}(t)\right)$, where $d \varrho^{(j)}(t)=\alpha^{(j)} \varrho^{(j)}(t) d t+W_{1}^{(j)}(t)$. The multiplicative term $\sigma_{u}(t)$ introduces a U-shape intraday volatility pattern. We follow Andersen et al. (2010b) and model $\sigma_{u}(t)$ according to Hasbrouck (1999) where,

$$
\sigma_{u}(t)=C+A \exp (-a t)+B \exp (-b(1-t)), \quad t \in[0,1],
$$

with $A=0.75, B=0.25, C=0.8893, a=10$ and $b=10$. As stated in Andersen et al. (2010b), these parameters mean that volatility at $t=0$ is three times the volatility at $t=0.5$ and volatility at $t=1$ is 1.5 times the volatility at $t=0.5$. The sizes of the (co)jumps are determined by $\sigma_{u}^{(j)}(t) \sigma^{(j)}(t) \psi(t)$, where we choose to define $\psi(t)=\kappa \times \phi(t)$ and $\phi(t)$ takes a value of -1 or 1 with equal probability. The innovation correlation (statistical leverage) for the continuous component of $p^{(j)}$ and $\varrho^{(j)}$ is given by $\rho^{(j)}$ and there is perfect correlation between the continuous components of the innovations in $\sigma^{(j)}(t)$ and $V^{(j)}(t)$. The correlation between the continuous components of $p^{(l)}$ and $p^{(m)}$ is $\sqrt{1-\left(\rho^{(l)}\right)^{2}} \sqrt{1-\left(\rho^{(m)}\right)^{2}}$.

We use the following parameter values $\left(\mu^{(j)}, \beta_{0}^{(j)}, \beta_{1}^{(j)}, \alpha^{(j)}, \lambda\right)=(0,-5 / 16,1 / 8,-1 / 40,0.1)$, for all $j$. Except for $\lambda$, these are identical to those used in Barndorff-Nielsen et al. (2011). However, for all $j$, we set $\rho^{(j)}=-0.83$, which corresponds to a correlation between the continuous components of all asset prices equal to 
$0.31^{8}$. We set $\kappa$ to take values of either $0, \sqrt{1 / 19}, \sqrt{1 / 9}, \sqrt{1 / 4}, \sqrt{3 / 7}$ or $\sqrt{2 / 3}$. These values correspond to (co)jumps contributing precisely $0 \%$ (no (co)jumps) or approximately $5 \%=\frac{\kappa^{2}}{1+\kappa^{2}}$ (very small (co)jumps), $10 \%, 20 \%$, $30 \%$ or $40 \%$ (very large (co)jumps) to QV on the day of a (co)jump arrival.

In order to simulate prices from (5), we use an Euler discretisation. We simulate prices every second for 55,000 days, where we assume there are 385 minutes in a day to match our empirical data. Hence, for each trading day, we simulate prices at times $t_{k}=k / K$, for $k=1, \ldots, K$ and $K=23,100$. The first 5,000 days are a burn-in period and are dropped from the final analyses. Since we are interested in the performance of the jump tests in a setting that reflects our empirical data, we sample (log) prices every 11 minutes. In addition, we introduce microstructure noise by adding an iid random variable to the $(\log )$ price so that we sample,

$$
y_{t, i}^{(j)}=p_{t, i}^{(j)}+e_{t, i}^{(j)}
$$

where $y_{t, i}^{(j)}$ is the $\log$ price of asset $j$ sampled on day $t$ at intraday time $i$, $e_{t, i}^{(j)} \sim \mathcal{N} \mathcal{I D}\left(0, \omega_{t, i}^{(j) 2}\right)$ and $\omega_{t, i}^{(j) 2}=0.001 \sum_{k=1}^{K}\left(\sigma^{(j)}\left(t_{k} / K\right)\right)^{2}$. A noise-to-signal ratio of 0.001 is consistent with the estimates in Hansen and Lunde (2006). Finally, we impose sparse sampling by randomly removing simulated prices such that the average time between observed prices is 5 seconds, i.e., we retain each one-second price with probability 0.2 .

\footnotetext{
${ }^{8}$ The mean correlation between asset prices in our sample is 0.25 . Although $\rho^{(j)}=-0.83$ leads to a very strong leverage effect, our results were unaffected if we set $\rho^{(j)}=0$ for all $j$ and let the continuous components of the log asset prices, $p^{j}$, be independent by replacing $W_{2}(t)$ by $W_{2}^{(j)}(t)$, where $d p^{(j)}(t)=\mu^{(j)} d t+\sigma_{u}(t)\left(d F^{(j)}(t)+\sigma^{(j)}(t) \psi(t) d N(t)\right)$ and $W_{2}^{(j)}(t)$ are independent.
} 


\subsection{Simulation Results}

Dumitru and Urga (2012) show that the ABD and LM tests are over-sized in finite samples, whereas the size of the BNS test is largely unaffected. Therefore, when applying the ABD and LM tests we apply the solutions proposed by Dumitru and Urga (2012). The first is to combine tests. Specifically,

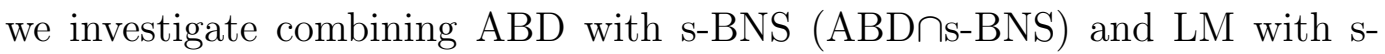

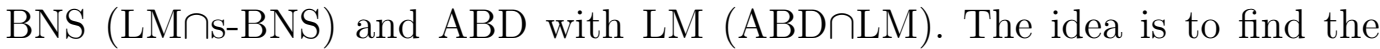
intersection between the jumps detected by the two tests and retain only the intersection of detected jumps. However, the effective sizes of the combined tests are far below the nominal significance levels applied to the ABD, LM and s-BNS tests individually.

The second solution is to adjust (reduce) the significance levels of the tests. For the ABD test, we find an appropriate size-adjustment can be obtained by simulating 50,000 days of $M$ normally distributed intraday returns with constant volatility. The adjusted significance level is selected such that the number of spurious jumps detected is consistent with the number expected under the nominal significance level ${ }^{9}$. In order to achieve an effective size of $1 \%$ with $M=35$, we find a significance level of $0.37 \%$ is required for the ABD test and $0.7 \%$ for the LM test.

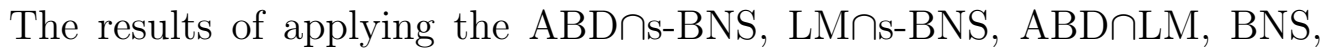
s-BNS and the size-adjusted ABD and LM detection methods are given in Table 1. For comparison, results from the BLT test are included. Note, to make the BLT results comparable to those of the other detection methods,

\footnotetext{
${ }^{9}$ Dumitru and Urga (2012) propose a slightly different solution. They recommend simulating 10,000 days of $M$ intraday returns at each intraday interval, where volatility is set equal to the volatility estimate for that interval. Using this method, critical values are updated for each intraday interval.
} 
we applied the test using a $0.0287 \%$ intraday significance level such that the daily significance level was $1 \%^{10}$. In addition, we include results from taking the intersection and union of jumps detected using all of the intraday detection methods, which include the size-adjusted ABD, size-adjusted LM, s-BNS, $\mathrm{ABD} \cap \mathrm{s}-\mathrm{BNS}, \mathrm{LM} \cap \mathrm{s}-\mathrm{BNS}$ and $\mathrm{ABD} \cap \mathrm{LM}^{11}$. We subsequently refer to these as the Intersection and Union detection methods, respectively. The rationale for including these detection methods is given by Dumitru and Urga (2012) and Schwert (2011) who show that different non-parametric jump tests do not agree on the timing of jump arrivals. This disparity is indicative of each test either detecting some jumps that cannot be detected by the other tests or of spurious jump detection. Dumitru and Urga (2012) suggest that by taking the union of the results from several tests, the power of jump detection can be improved, albeit at the expense of higher power. Conversely, they suggest that taking the intersection of the results will minimise spurious jump detection. Thus, the Intersection and Union methods should, respectively, minimise spurious cojump detection and maximise the power of cojump detection.

Panel A of Table 1 reports the effective sizes of the detection methods when used to detect jumps in a univariate series. In Panel B we report the effective sizes and effective powers of the cojump detection methods as well as the median number of stocks detected to be involved in each cojump. Note, the effective sizes are reported as daily percentages, i.e., they represent the percentage of days on which a spurious (co)jump was detected. The effective

\footnotetext{
${ }^{10}$ This is the same Bonferroni adjustment that is made for the ABD test $\left(1-(1-\alpha)^{\frac{1}{M}}\right)$.

${ }^{11}$ Note, there is no size adjustment applied to the ABD or LM tests when they are

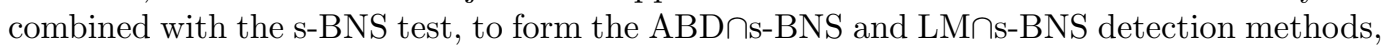

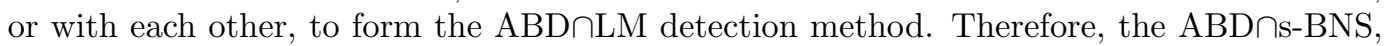
$\mathrm{LM} \cap \mathrm{s}-\mathrm{BNS}$ and $\mathrm{ABD} \cap \mathrm{LM}$ detection methods may lead to results that differ to those that would be obtained if the size-adjusted ABD and size-adjusted LM tests are combined with the s-BNS test and each other.
} 
power represents the percentage of true cojumps correctly detected. Results for different sizes of (co)jump, i.e., different values of $\kappa$, are given in Sub-Panels I-VI.

From Panel A of Table 1 it can be seen that the size-adjustments applied to the ABD and LM tests work well. Their effective sizes are close to the $1 \%$ significance level. The effect of taking intersections and unions of the jump

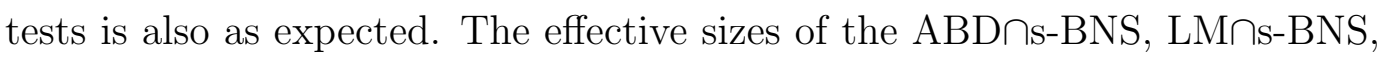
$\mathrm{ABD} \cap \mathrm{LM}$ and Intersection detection methods are below the $1 \%$ significance level used in each test, whereas the effective size of the Union detection method is $>2 \%$, which is relatively high.

Sub-Panel I of Panel B in Table 1 leads to similar qualitative conclusions about the effective sizes of the cojump detection methods. However, it seems that aggregating jumps from the univariate jump tests via the coexceedance criterion to detect cojumps results in random spurious jumps being aggregated to produce a reasonable number of spurious cojumps. Hence, the effective sizes of cojump detection are larger than the effective sizes of jump detection in the univariate series and are particularly large for the BNS and Union detection methods. However, the number of stocks detected to be involved in these spurious cojumps is generally small, with the median being 2 stocks. Thus, the coexceedance based cojump detection methods may lead to a reasonable number of spurious cojumps being detected, but the number of stocks involved in these will be very low. Note, the effective size of the BLT test is close to its nominal daily significance level of $1 \%$.

Sub-Panels II-VI of Panel B in Table 1 show that, as expected, the effective powers of all the detection methods, including the BLT test, decrease as the size of the (co)jumps decreases. However, the two most interesting observations are as follows. Firstly, the effective powers of the coexceedance based 
detection methods are similar to, and often higher, than the BLT test for all sizes of (co)jump. Although, the quid-pro-quo is higher effective sizes when using the coexceedance based detection methods. Secondly, one should expect to detect only a fraction of the stocks involved in a cojump when using the coexceedance based detection methods. This is true for all sizes of (co)jump.

For example, when $\kappa=\sqrt{1 / 4}$ so that we expect the (co)jumps to be responsible for a non-trivial 20\%, approximately, of the QV in each stock on (co)jump days, we expect to detect around $1 / 6$ of the stocks in the sample to be involved. Even when the (co)jumps are very large $(\kappa=\sqrt{2 / 3})$, we would only expect to detect around $2 / 3$ of the stocks in the sample to be involved.

\section{Data}

We obtained high-frequency transaction data from the trades and quotes (TAQ) database for the period January 2002 to June 2011. We sampled prices with time stamps falling within 09:35-16:00 EST. The first 5 minutes of the trading day, which officially starts at 09:30 EST, is ignored due to the potentially erratic price behaviour induced by the market opening procedure. Appendix A explains our data cleaning methods. Our sample consists of 60 liquid stocks and a proxy for the market portfolio, the Spider ETF (SPY), which replicates the S\&P 500. Appendix B explains our sample selection procedure.

Although theoretically the accuracy of RV and BV estimates increases as the sampling interval over which intraday returns are measured decreases, empirically such a relationship is limited by the presence of microstructure noise, such as bid-ask bounce, price discreteness and irregular trading. These bias the RV and BV estimates, with the effects increasing in magnitude as the time interval over which intraday returns are sampled decreases. Using 
volatility signature plots (Andersen et al., 2000), we decide to sample prices over intraday intervals of 11 minutes $(M=35)^{12}$.

\section{Intraday Jumps}

Before examining cojumps, we summarise results from applying our jump tests to the individual securities. To provide robustness against a potentially time-varying intraday volatility pattern, we follow Lahaye et al. (2011) and estimate the WSD intraday volatility correctors for non-overlapping windows of six months of data. We use a daily $1 \%$ significance level in all jump tests. Analogous results are obtained using either a $5 \%$ or $0.1 \%$ significance level (available on request).

Table 2 presents the mean number of jumps detected by each detection method as well as the mean number and size (absolute values) of positive and negative standardised jumps detected by the intraday jump tests ${ }^{13}$. Note, each test detects a larger number of jumps than the 24 jumps we would expect from

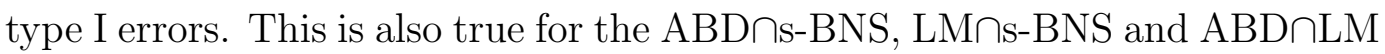
tests, which have an effective size much lower than $1 \%$.

Table 2 is also informative with respect to some of the jump properties. In particular, it shows that, on average, the number and mean sizes of positive and negative jumps detected for each security are similar. To test for significant differences between the number and sizes of positive and negative jumps, we conducted Chi-squared goodness-of-fit tests and Wilcoxon rank sum tests, respectively ${ }^{14}$. Table 2 shows few significant differences were identified

\footnotetext{
${ }^{12}$ The volatility signature plots and a list of the selected firms are available on request.

${ }^{13}$ The size of a standardised jump is given by $\frac{\left|r_{t, i}\right|}{\sqrt{\Delta \cdot B V_{t} \cdot s_{W S D, i}^{2}}}$.

${ }^{14}$ Taking absolute values means the distributions of positive and negative jump sizes are non-normal. We therefore preferred the Wilcoxon rank sum test over two-sample t-tests.
} 
in either case. Therefore, the detected jumps appear to be symmetrically distributed. This supports Lee and Mykland (2008) and Lahaye et al. (2011) who report similar findings of symmetrically distributed jumps for individual stocks, stock index futures, T-bond futures and exchange rates.

\section{Cojump Results}

We report results from our six intraday detection methods, the Intersection and Union detection methods, the BNS test (our daily detection method) and the BLT test. All results are obtained using a daily 1\% significance level and the SPY is used to proxy the market portfolio. Analogous results are found when either a $5 \%$ or $0.1 \%$ significance level is employed and when using high-frequency data sampled at either 17.5 minutes or 5 minutes, but are not included to conserve space.

\subsection{Comparison Across Detection Methods}

In Table 3 we report the average numbers of cojumps and cojump days, the median numbers of stocks found to be involved in the cojumps and the mean proportions of jumps detected in the individual stocks which are involved in a cojump. Panel A summarises the results for all cojumps, whilst Panels $\mathrm{B}$ and $\mathrm{C}$ summarise the results for systematic and non-systematic cojumps, respectively.

Table 3 shows that both systematic and non-systematic cojumps were detected by all the detection methods. The lower number of cojumps (systematic and non-systematic) and the lower proportion of individual stock jumps in-

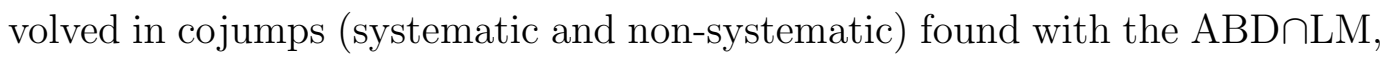
$\mathrm{ABD} \cap \mathrm{s}-\mathrm{BNS}, \mathrm{LM} \cap \mathrm{s}-\mathrm{BNS}$ and Intersection detection methods is due to the effective sizes of these detection methods being much lower than those of the 
other detection methods. The much lower (higher) number of cojumps detected by the Intersection (Union) method is indicative of disparity between cojumps detected by the alternative detection methods. This highlights the importance of using multiple (co)jump tests; in order to detect a larger proportion of the common jumps, it may be necessary to aggregate results from the alternative detection methods, i.e., to use the Union detection method.

The importance of using intraday detection methods is highlighted by comparing the results from the (daily) BNS test to those from the s-BNS and other intraday detection methods. The BNS test detects cojumps on many more days and the proportion of individual stock jumps involved in cojumps is higher compared to the cojumps detected by the s-BNS and other intraday detection methods ${ }^{15}$. This suggests many of the daily cojumps detected by the BNS test are disjoint cojumps. Specifically, jumps are occurring on the same day for different stocks, but at different times within the day.

\subsection{Association between Market Jumps and Cojumps between Stocks}

Table 3 shows that, across all detection methods, a majority of cojumps are non-systematic, with a relatively low proportion $(<18 \%)$ of individual stock jumps being involved in systematic cojumps. However, the median number of stocks involved in systematic cojumps is significantly higher than the median number involved in non-systematic cojumps for most detection methods. This supports Hypothesis 1; there appears to be an association between jumps in the market portfolio and cojumps among the underlying stocks.

\footnotetext{
${ }^{15}$ Panel A of Table 3 also shows the median number of stocks detected to be involved in cojumps is higher for the BNS test (5 stocks) compared to the s-BNS and other detection methods (2 stocks). Although this does not appear to be a large difference, the number of stocks involved in the cojumps detected by the BNS test is, generally, much larger than the numbers detected to be involved by the s-BNS and other intraday detection methods.
} 
Further support for Hypothesis 1 is provided by running Poisson count regressions. Specifically, we regress the number of stocks detected to be involved in a cojump $\left(C O U N T_{t, i}\right)$ against an indicator which takes a value of 1 when a jump is detected in our market proxy and 0 otherwise $\left(I S P Y_{t, i}\right)$. Since this is a Poisson regression, the conditional mean of $\ln C O U N T_{t, i}$ is linear in the indicator variable ${ }^{16}$,

$$
\ln \mu_{t, i}=E\left[\ln C O U N T_{t, i} \mid I S P Y_{t, i}\right]=\beta_{o}+\beta_{1} I S P Y_{t, i},
$$

and

$$
P\left[C O U N T_{t, i}=n \mid I S P Y_{t, i}\right]=\frac{e^{-\mu_{t, i}} \mu_{t, i}^{n}}{n !} .
$$

The Poisson distribution assumes the (conditional) mean and variance are equal. This very restrictive assumption most likely does not apply to our cojumps. Therefore, the parameters are estimated by the Method of Moments (see Cameron and Trivedi (1998)) which results in standard errors that are robust to misspecification of the distribution of $C O U N T_{t, i}{ }^{17}$.

\footnotetext{
${ }^{16}$ Since $I S P Y_{t, i}$ is an indicator variable, $C O U N T_{t, i}$ is also linear in $I S P Y_{t, i}$.

${ }^{17}$ Note, the sample moment conditions are $\sum_{t=1}^{T} \sum_{i=1}^{M}\left(y_{t, i}-\mu_{t, i}\right) \mathbf{x}_{t, i}=\mathbf{0}$, where $y_{t, i}=$
} $C O U N T_{t, i}$ and $\mathbf{x}_{t, i}=\left(1, I S P Y_{t, i}\right)^{\prime}$. Although these moment conditions are identical to the first-order conditions for the Poisson MLE, the standard errors are given by $\operatorname{Var}(\hat{\boldsymbol{\beta}})=$ $\left(\sum_{t=1}^{T} \sum_{i=1}^{M} \mu_{t, i} \mathbf{x}_{i} \mathbf{x}_{i}^{\prime}\right)^{-1}\left(\sum_{t=1}^{T} \sum_{i=1}^{M}\left(y_{t, i}-\mu_{t, i}\right)^{2} \mathbf{x}_{i} \mathbf{x}_{i}^{\prime}\right)\left(\sum_{t=1}^{T} \sum_{i=1}^{M} \mu_{t, i} \mathbf{x}_{i} \mathbf{x}_{i}^{\prime}\right)^{-1}$, where $\hat{\boldsymbol{\beta}}=\left(\hat{\beta}_{0}, \hat{\beta}_{1}\right)^{\prime}$. This estimator of the standard errors is referred to as the robust sandwich estimator by Cameron and Trivedi (1998). It is robust to misspecification of the distribution of $C O U N T_{t, i}$. In particular, it is consistent if the conditional mean and conditional variance of $C O U N T_{t, i}$ are not equal. Nevertheless, as a robustness check, we also ran count regressions which assumed $C O U N T_{t, i}$ followed a negative binomial distribution, a zero-inflated Poisson and a zero-inflated negative binomial distribution. All estimated parameters were similar and there were no qualitative differences in the conclusions regarding the significance of parameters. 
Parameter estimates, in which $C O U N T_{t, i}$ and $I S P Y_{t, i}$ are determined by each of our detection methods, are given in Panel A of Table 4. Estimates of $\beta_{1}$ are significant at the $0.1 \%$ level in all cases, showing there is a significant relationship between jumps in the market portfolio proxy and cojumps amongst the stocks. Although there is a significant relationship, the number of stocks expected to cojump conditional on a jump in the market proxy is moderate compared to the size of our sample in all cases. Similarly, the median numbers of stocks involved in cojumps reported in Table 3 are moderate relative to the number of stocks in our sample.

However, our Monte Carlo simulations in Section 3 demonstrated that it is difficult to detect all common jumps comprising a cojump when using the coexceedance based detection methods. This was true whether the common jumps were large or small. Hence, although a moderate proportion of stocks were detected to be involved in the systematic cojumps, we believe they represent true systematic cojumps and that more stocks (if not all) are involved in the systematic cojumps.

This finding differs to BLT, who argued that there is almost no evidence for a contemporaneous association between jumps in the market portfolio and jumps in the underlying stocks, i.e., systematic cojumps, when using the coexceedance approach with the BNS test. BLT suggest that cojumps between individual stocks and the market portfolio do occur, but that the jumps involved tend to be small and cannot be detected using univariate jump tests. It should be reiterated that the terms "large jump" and "small jump" are relative. A jump is detectable (undetectable) if it is large (small) relative to the instantaneous volatility of the continuous component of price changes $(\sigma(t)$ in eq. (1)). Thus, due to instantaneous volatility being stochastic, a jump of given magnitude $(\kappa(t)$ in eq. (1)) may be large and detectable at one point 
in time, but small and undetectable at another. BLT argue that systematic cojumps are masked by additional volatility introduced by idiosyncratic price changes in the individual stocks.

Specifically, BLT find almost no correlation between $Z_{B N S, t}$ statistics for their market proxy and the individual stocks, but do not report the number of stocks involved in cojumps. For comparison, in Panel B of Table 4 we report the mean correlation between ABD, LM and BNS test statistics for individual stocks and the SPY. We also report the mean values of estimated intercept $\left(\beta_{0}\right)$ and slope parameters $\left(\beta_{1}\right)$ from univariate regressions of ABD, LM and BNS test statistics for individual stocks against the corresponding test statistics for the SPY. It can be seen that the correlations and slope parameters are indeed low on average, which could lead to the conclusion that there is no association between jumps in the market portfolio proxy and cojumps in the stocks. However, the estimated parameters are significant in nearly all cases.

The results of BLT have two implications. Firstly, their results suggest systematic cojumps tend to be associated with small jumps in individual stocks. Secondly, their results are consistent with large jumps in individual stocks being primarily generated by firm-specific news. In contrast, by examining the number of jumps detected to be involved in the cojumps, we argue that it is possible to detect cojumps using coexceedance based detection methods, although one should not expect to detect common jumps in all stocks. Furthermore, our results suggest systematic cojumps may be associated with large jumps in individual stocks and, therefore, that market-level news is important in generating simultaneous large jumps in individual stocks.

The fact that systematic cojumps may be comprised of large common jumps in the individual stocks and that it is unlikely all of the common jumps will be identified by the coexceedance based detection methods also has impor- 
tant implications for understanding the dependence between the probability distributions governing jumps. For example, the tail dependence metrics developed in Bollerslev et al. (2013) rely on identifying systematic cojumps in bivariate series of the market portfolio and an individual stock, where the common jumps are large. Bollerslev et al. (2013) use a coexceedance based detection method to identify these cojumps. Our results suggest that large systematic (co)jumps exist, but that not all of them will be detected if only bivariate series are used in a coexceedance based detection method.

\subsection{Comparison of the Number of Stocks Involved in Cojumps}

Given that the systematic cojumps are found to involve relatively small numbers of stocks, we consider the possibility that these numbers arise by chance or, in other words, that the jumps in the stocks are independent. Table 5 and Figure 1 summarise the number and proportions of systematic and non-systematic cojumps involving different numbers of stocks. We shall refer to these as the systematic and non-systematic cojump distributions. It is easier to read the numbers of systematic and non-systematic cojumps in Table 5, whilst Figure 1 provides a more intuitive visual representation of the distributions. In contrast, Figure 2 plots the distribution we would expect if all jump arrivals in the underlying stocks and the market index were independent. The cojump distribution in Figure 2 follows a Poisson-Binomial probability distribution (see Chen and Liu (1997)).

The Poisson-Binomial distribution is defined as follows. Let $\left(Z_{1}, \ldots, Z_{N}\right)$ be a vector of independent Bernoulli random variables which respectively take a value of 1 with probability $\left(p_{1}, \ldots, p_{N}\right)$ and 0 otherwise. Then $S_{Z}=Z_{1}+$ $\cdots+Z_{N}$ is a random variable which follows a Poisson-Binomial distribution and 
may take values from $\{0, \ldots, N\}^{18}$. Specifically, the probability distribution is given by,

$$
P\left(S_{Z}=n\right)=\left(\prod_{i=1}^{N}\left(1-p_{i}\right)\right) \sum_{i_{1}<\cdots<i_{n}} w_{i_{1}} \ldots w_{i_{n}}
$$

where $w_{i}=p_{i} /\left(1-p_{i}\right)$ and $i=1, \ldots, N$. Note, the summation is over all combinations $(N ! / n !(N-n) !)$ of $i_{1}, \ldots, i_{n}$ from $\{1, \ldots, N\}$. In our application $p_{i}$ is the probability of a jump in stock $i$ and $P\left(S_{Z}=n\right)$ is the probability that $n$ stocks participate in a cojump. We estimate values of $p_{i}$ by taking the ratio of detected jumps in each stock to the total number of time periods in the sample, i.e. $\hat{p}_{i}=$ Number of detected jumps for stock $i$ /Total number of intraday intervals.

Several observations should be noted. Firstly, as expected, the proportions of systematic and non-systematic cojumps involving different numbers of stocks differ between detection methods due to their differing effective sizes and powers. Secondly, there is a very clear difference between the systematic and non-systematic cojump distributions for each detection method. Although each detection method detects a relatively large proportion of systematic cojumps which involve a moderate number of stocks, it is seen clearly that systematic cojumps involve more stocks than non-systematic cojumps. This is the same evidence supporting Hypothesis 1 documented in Section 6.2, but presented in an alternative way. Furthermore, since we detect $24.5 \%-35.9 \%$ of our systematic cojumps to involve more than 10 stocks with the ABD, LM, $\mathrm{ABD} \cap \mathrm{LM}$ and Union detection methods, we believe there is evidence for an association between large jumps in the market proxy and large jumps in the

\footnotetext{
${ }^{18}$ When $p_{1}=p_{2}=\cdots=p_{N}$, the Poisson-Binomial distribution reduces to the binomial distribution.
} 
underlying stocks. The lower proportions of systematic cojumps involving more than 10 stocks for the remaining detection methods, $6.8 \%-7.4 \%$, reflect their lower effective sizes and powers.

Lastly, the visual contrast between the systematic cojump distribution and the distribution we would expect if jumps were independent is striking and supports our conjecture that the systematic cojumps we detect are true cojumps. This is also supported by formal tests. The p-values associated with Chi-squared and Kolmogorov-Smirnov tests are $<0.001$ when we compare the systematic (co)jump and independent (co)jump distributions for each detection method ${ }^{19}$.

In contrast, it appears the many non-systematic cojumps which involve two or three stocks may arise by chance; there is no discernible difference between the independent jump distribution and the non-systematic cojump distribution. This is consistent with our Monte Carlo simulation results which showed that a reasonable number of spurious cojumps involving low numbers of stocks may be detected with the coexceedance based detection methods. However, the p-values associated with Chi-squared and Kolmogorov-Smirnov tests are $<0.001$ when we compare the non-systematic (co)jump and independent (co)jump distributions for each detection method. This is probably due to the non-systematic cojumps we detect which involve a relatively large number of stocks. Although these cojumps represent a small proportion of non-systematic cojumps, there is still a relatively large number of them. For

\footnotetext{
${ }^{19}$ We do not correct the asymptotic distribution in the Chi-squared test for the parameters we had to estimate in order to obtain the Poisson-Binomial distribution for the independent (co)jumps. However, as the p-values are so small, we do not believe this has an impact on our conclusions. Similarly, we do not correct the Kolmogorov-Smirnov test to take account of the fact that the (co)jump distributions are discrete rather than continuous. However, failing to take this into account leads to conservative p-values (see Conover (1972)).
} 
example, across the detection methods, there are 1 to 53 non-systematic cojumps that involve more than 10 stocks.

\subsection{Using the EQW as the Market Portfolio Proxy}

In Table 5 it can be seen that a relatively large proportion of systematic cojumps involve five or fewer stocks and we also detect singular SPY jumps. One potential explanation is that our market proxy, the SPY, is not perfectly linked to the stocks included in our sample. It is possible that some of the singular SPY jumps are spurious or not linked to the underlying stocks because the SPY has its own price dynamics. Similarly, the sample of 60 stocks is only a fraction of the S\&P 500. Stocks in the S\&P 500 excluded from our sample could be involved in cojumps with the SPY, leading to the detection of singular jumps in the SPY. To establish a perfect link between jumps in the market proxy and jumps in the prices of the underlying stocks, we follow Bollerslev et al. (2008) and replace the SPY with an equally-weighted portfolio of the 60 stocks in our sample (EQW). In the following, we use the prefixes EQWand SPY- to highlight when we are referring to systematic and non-systematic cojumps identified using the EQW or SPY market proxies.

The number of EQW-systematic and EQW-non-systematic cojumps involving different numbers of stocks are given in Table 6. Establishing a perfect link clearly helps explain singular jumps in the market proxy. Compared to SPY-systematic jumps, lower numbers of singular EQW-systematic jumps are detected and the numbers of EQW-systematic cojumps involving more than 10 stocks are all higher, except for the Intersection detection method. In contrast, there is little difference between the results for EQW-non-systematic cojumps and SPY-non-systematic cojumps. A non-trivial number of non-systematic cojumps continue to involve relatively large numbers of stocks. 


\subsection{Small Jumps in the Market Portfolio}

One reason for detecting non-systematic cojumps that involve a large number of stocks might be because they occur concomitantly with small, undetected, jumps in the market proxy. To investigate the likelihood of a type II error in the detection of a small jump in the market proxy when a large number of stocks are found to be involved in a non-systematic cojump, we calculate the p-values of the ABD and LM tests applied to the SPY and examine whether non-systematic cojumps are associated with low p-values. Small pvalues, slightly larger than the significance level of $1 \%$, would be indicative of type II errors.

Table 7 summarises the number of non-systematic cojumps and the maximum, median and minimum number of stocks detected to be involved in the non-systematic cojumps when the p-value for the SPY is less than $5 \%$ and $10 \%$. We also report the number of non-systematic cojumps associated with these p-values in the SPY which involve more than 10 stocks, where we consider 10 or more stocks to be a large number. Panel A summarises results when p-values are computed using the ABD test and Panel B summarises results when p-values are computed using the LM test. It can be seen from both panels that there is some evidence of non-systematic cojumps involving large numbers of stocks being associated with type II errors in the detection of jumps in the market portfolio proxy. By comparing Table 7 with Panel B of Table 5 , it can be seen that many of the non-systematic cojumps detected to involve more than 10 stocks are associated with p-values in the SPY which are less than $5 \%^{20}$. Furthermore, for many of the detection methods, non-

\footnotetext{
${ }^{20}$ Note, the jumps in the individual stocks comprising the non-systematic cojumps which involve more than 10 stocks are all of the same sign. That is, all stocks are cojumping in the same direction. This lends further support to our conjecture that these non-systematic
} 
systematic cojumps involving the maximum number of stocks are associated with a p-value for the SPY which is less than $5 \%$.

\subsection{Non-systematic Cojumps}

After taking into consideration the non-systematic cojumps that are likely to have been misclassified, there remain some that involve relatively large numbers of stocks. We explore two further possible explanations. The first is that they represent industry cojumps. If all the stocks involved in a nonsystematic cojump are from the same industry, then we refer to the nonsystematic cojump as an industry cojump. The second is that the cojumps could be a result of sensitivity to other risk factors. In particular, we explore whether these cojumps occur concomitantly with jumps in proxies for the FF SMB and HML factors (Fama and French, 1992, 1993). To conserve space, we use results from our Union detection method and use the SPY as the market proxy only.

To examine industry cojumps, the stocks were sorted into industry portfolios by SIC code. We used the industry allocations available from the website of Kenneth R. French ${ }^{21}$. Below, we present results based on sorting the stocks into 12 industries. Note, our conclusions do not change if we allocate the stocks into 5, 10 or 17 industries.

In Panel A of Table 8 we report the number of stocks belonging to each of the 12 industries, the number of industry cojumps detected and the maximum, median and minimum number of stocks detected to be involved in each type of industry cojump. It can be seen that our sample of stocks is concentrated in

cojumps are misclassified. If the stocks were cojumping in opposite directions, then it would be more likely that these non-systematic cojumps are true diversifiable non-systematic cojumps.

${ }^{21}$ http://mba.tuck.dartmouth.edu/pages/faculty/ken.french/. 
the Business Equipment industry. Thus, some of our non-systematic cojumps which involve relatively large numbers of stocks might be explained by cojumps occurring exclusively amongst the stocks within this industry. However, we do not find any evidence for this. The number of stocks involved in the Business Equipment industry cojumps tends to be low ( $<8$ stocks), with many of the cojumps involving just 2 stocks. The membership of stocks to the remaining industries is relatively low so that cojumps in these industries cannot explain non-systematic cojumps involving large numbers of stocks ${ }^{22}$.

In order to construct FF factor proxies, we follow Bannouh et al. (2010) in using intraday returns for ETFs that proxy small capitalisation, value and growth portfolios. Specifically, the SMB mimicking portfolio is formed by taking returns from the SPY and subtracting returns from the iShares Russell 2000 Index Fund (IWM). The HML mimicking portfolio is formed by taking returns on the iShares S\&P 500 Value Index Fund (IVE) and subtracting returns from the iShares S\&P 500 Growth Index Fund (IVW). Panel B of Table 8 reports the number of cojumps between the SMB and HML factors and the stocks. To ensure we isolate the influence from the FF factors, the FF cojumps reported exclude times where the SMB and HML factors cojump with the SPY. However, we do report cojumps in which both the SMB and HML factors jump. We report the maximum, median and minimum number of stocks detected to be involved in the FF cojumps.

From Panel B of Table 8 it can be seen that we do not find that cojumps with the FF factor proxies explain non-systematic cojumps involving large numbers of stocks. In general, the number of stocks involved in the FF cojumps

\footnotetext{
${ }^{22}$ Bajgrowicz and Scaillet (2011) find industry cojumps amongst the 30 DJIA stocks. However, their results are based exclusively on the BNS test and they group stocks using Global Industry Classification Standard (GICS) codes.
} 
is low. Although, it is interesting to note that there may be some true FF cojumps which occur independently of cojumps with the market portfolio ${ }^{23}$.

\subsection{Comparison with Bollerslev, Law and Tauchen (2008)}

In this section we examine whether our coexceedance based detection methods and the BLT test are substitute approaches to cojump detection. We are therefore interested in the degree to which the timings of the cojumps detected by the BLT test and the coexceedance based detection methods coincide. A high degree of coincidence, such that the BLT test and coexceedance based detection methods detect cojumps at identical times, would suggest the alternative detection methods are substitutes for one another. In contrast, a low degree of coincidence, such that the BLT test and coexceedance based detection methods detect cojumps at largely differing times, would suggest that combining the tests may improve the power of cojump detection.

Our Monte Carlo simulations showed that the powers of the coexceedance based detection methods and the BLT test are similar. This suggests we should expect at least some coincidence in the timing of cojump detection. However, we have also demonstrated that taking the union of cojumps detected using the coexceedance based detection methods may improve the power of cojump detection. We therefore expect there to be some benefit in combining the coexceedance based detection methods and the BLT test.

In Panel A of Table 9 we document the intersections between cojumps detected by the BLT test and each of our coexceedance based detection methods. Since we have provided evidence of a tendency for cojumps to occur when the market jumps, we expect there to be a higher incidence of intersection with the

\footnotetext{
${ }^{23}$ Of course, this analysis could be extended by considering alternative industry allocations and proxies for the FF factors. However, this is beyond the scope of the paper.
} 
BLT test when examining systematic cojumps. Therefore, we also provide a breakdown of the results into intersections with systematic and non-systematic cojumps in Panels B-C of Table 9. The SPY is used as the market proxy in all cases. Analogous results are obtained if EQW is used, but are not reported to conserve space.

The results in Panels A-C of Table 9 are as expected. Many of the cojumps detected by the BLT test, between $22 \%$ and $90 \%$, are also detected by our coexceedance based detection methods. There is also a relatively high degree of association between systematic cojumps detected with our coexceedance based detection methods and the BLT cojumps, where between $18 \%$ and $54 \%$ of the systematic cojumps are also detected by the BLT test. Although, it is surprising that larger proportions of the systematic cojumps are not also detected by the BLT test.

The majority of intersections between the coexceedance based detection methods and the BLT test are with non-systematic cojumps. It is noteworthy that there are intersections with non-systematic cojumps that involve relatively large numbers of stocks. This is demonstrated by the maximum number of stocks involved in the non-systematic cojumps intersected by the BLT test being between 11 and 32 stocks. This lends further support to our conjecture that some non-systematic cojumps are misclassified. However, there are also BLT cojumps that do not intersect with any cojumps detected by the coexceedance based detection methods.

On balance, the results in this Section suggest that the coexceedance based detection methods and the BLT test could be employed in a similar manner to that recommended by Dumitru and Urga (2012) for univariate jump tests. If we would like to be more certain that they are detecting true cojumps, then we could only select cojumps detected by both the coexceedance based detection 
methods and the BLT test. On the other hand, if we would like to maximise the power of cojump detection, then we could select cojumps detected by either the coexceedance based detection methods or the BLT test. The effect on the number of systematic cojumps detected would be substantial in either case (assuming cojumps detected by the BLT test are systematic). This is demonstrated in Table 10, which reports the number of systematic cojumps we would detect if we select only those intersected by the BLT test and the number we would detect if we select systematic cojumps detected by either the BLT test or the coexceedance based detection methods.

\section{Association between Economic News and Cojumps}

\subsection{Macroeconomic News}

Our analysis of Hypothesis 2 is based on cojumps detected using the Union detection method ${ }^{24}$. A total of 6 regularly scheduled macroeconomic news announcements, which are released during trading hours (09:30-16:00), were included in the analysis. These are for Construction Spending, Factory Orders, ISM Report on Business and New Home Sales, all released at 10:00; the Federal Funds Target Rate, released at 14:15; and Consumer Credit, released at 15:00. Although a full analysis should take into consideration the surprise component of news announcements, this is beyond the scope of our paper. We therefore examine the relationship between the timing of detected (co)jumps and the release times of the macroeconomic news announcements.

We follow Lahaye et al. (2011) and report in Table 11 estimates, for all, systematic and non-systematic (co)jumps, of the probabilities of (co)jump ar-

\footnotetext{
${ }^{24}$ Analogous results hold when the $0.1 \%$ and $5 \%$ significance levels are adopted and other detection methods are used.
} 
rivals conditioned on the release of macroeconomic news. We also report the numbers of (co)jumps detected to coincide with the release of the macroeconomic news announcements and the mean number of stocks involved in each type of (co)jump. For comparison, we also report this information conditioning only on intraday time. In other words, we report this information for (co)jumps arriving during the 09:57-10:08, 14:10-14:21 and 14:54-15:05 intraday intervals, which straddle the macroeconomic news announcement times, without conditioning on a macroeconomic news announcement.

From Table 11 it can be seen that conditioning on the release of macroeconomic news more than doubles the probability of a (co)jump arrival. However, the number of stocks detected to be involved in the (co)jumps increases substantially for the Federal Funds Target Rate announcement only. When the (co)jumps are separated into systematic and non-systematic (co)jumps, it can be seen that conditioning on the release of macroeconomic news leads to a large relative increase in the probability of either type of (co)jump. Surprisingly, the conditional probability of a non-systematic cojump is higher than a systematic cojump. Indeed, the numbers of systematic (co)jumps found to be associated with the release of macroeconomic news are low (except, arguably, for those associated with news released about the Federal Funds Target Rate). However, the mean number of stocks involved in non-systematic (co)jumps associated with the release of macroeconomic news is under 2 (except for the Federal Funds Target Rate), suggesting that many are singular jumps. Therefore, it is unlikely that a causal effect exists between the release of macroeconomic news and the occurrence of many of the non-systematic (co)jumps. In comparison, the number of stocks involved in systematic (co)jumps associated with the release of macroeconomic news is large, especially when compared to the results in Table 5. 
The results in Table 11 suggest there is a particularly strong relationship between Federal Funds Target Rate announcements and the arrival of systematic (co)jumps. This announcement is associated with the largest probability of a systematic (co)jump and the largest number of stocks involved in systematic (co)jumps. The same is true of non-systematic (co)jumps, which, again, suggests some non-systematic (co)jumps may in fact be systematic. This evidence is consistent with Lahaye et al. (2011), Bajgrowicz and Scaillet (2011) and Lee (2012) who also find a strong association between jumps in equity markets and Federal Funds Target Rate news announcements.

\subsection{Systematic Cojumps and Other News}

Since macroeconomic news announcements do not explain all systematic cojumps, we examine whether they are associated with other news reported in press articles. We selected a sub-sample of systematic cojumps, which were not associated with macroeconomic news announcements, involving the ten largest numbers of stocks detected by the Union coexceedance detection method. We obtained relevant news articles from the Nexis database. A summary of the dates, numbers of stocks involved, the news identified and the sign of the jumps in the individual stocks comprising the systematic cojumps is given in Table 12. Note, the sign of the jumps in the individual stocks comprising the systematic cojumps were identical in all cases and that all information related to relevant news is paraphrased from The Associated Press newswire. In most cases, some important news could be identified. Of course, we cannot say with certainty whether the news was directly responsible for the systematic cojump, although there appears to be a good match between the description

in the press and our systematic cojumps. In addition, the signs of the jumps in the individual stocks comprising the systematic cojumps are consistent with 
the reaction that would be expected from the corresponding news.

However, on 5 February 2010 there was very little reported in the way of abnormal price changes, yet we detected a systematic cojump involving 33 stocks. The Flash Crash of 6 May 2010 is another contentious example. Christensen et al. (2011) have recently argued this event can be explained by a volatility burst. Given the unusual circumstances surrounding this event, such as the role of algorithmic trading (for example, see Easley et al. (2011)), it is difficult to conclude with certainty whether the event should be attributed to a price jump or a volatility burst. Needless to say, further research on the Flash Crash and the volatility burst hypothesis is required.

\section{Conclusions}

In this paper we investigate cojumps in stock prices. We examine two hypotheses. The first is that there should be an association between jumps in the market portfolio and common jumps, or cojumps, among the underlying stocks (Hypothesis 1). We refer to cojumps which involve the market portfolio as systematic cojumps, since they represent non-diversifiable cojumps. The second is that systematic cojumps will be associated with the release of macroeconomic news (Hypothesis 2). Since macroeconomic news can be considered to have a market-level influence, it is an obvious candidate as a source of systematic cojumps.

We detect cojumps by applying non-parametric intraday jump tests to a panel of high-frequency stock prices. Cojumps are then detected via a coexceedance criterion. We refer to cojump detection methods adopting this procedure as coexceedance based detection methods. We also apply the intraday non-parametric cojump test developed by BLT, which does not rely on a coexceedance criterion. 
Our first contribution is to show, through a Monte Carlo simulation study, that it is possible to detect cojumps using the coexceedance based detection methods. We show that the powers of the coexceedance based detection methods are similar to the BLT test. Importantly, we also show that it is unlikely that all the common jumps comprising a cojump will be detected by the coexceedance based detection methods, irrespective of whether the (co)jumps are large or small. This is in contrast to BLT, who argue it is not possible to detect cojumps in stock prices using such coexceedance based detection methods and that their cojump test is more powerful in detecting small (co)jumps.

Our second contribution is to document empirical evidence supporting Hypothesis 1 . The number of stocks we detect to be involved in the systematic cojumps is moderate, but, given the results from our Monte Carlo simulation study, we argue that we detect true systematic cojumps. BLT argue that it is not possible to detect cojumps in stock prices using coexceedance based detection methods because the jumps comprising a systematic cojump are small and undetectable and that firm-specific news is primarily responsible for generating large jumps in stock prices. In contrast, our results suggest that a systematic cojump may involve large jumps in individual stocks and that market-level news is able to generate simultaneous large jumps in stock prices.

In addition, we detect non-systematic cojumps which involve relatively large numbers of stocks. We are unable to explain these as being a consequence of industry cojumps or cojumps associated with the FF SMB and HML risk factors. Hence, we believe more systematic cojumps occur than is suggested by the jumps detected in the proxy for the market portfolio. This is in agreement with the conjecture made by BLT based on the results from their cojump test. We also compare empirical results obtained from our coexceedance based detection methods to those obtained from the BLT test. In analogy with 
Dumitru and Urga (2012) for univariate jump tests, we suggest that spurious cojump detection can be minimised by taking the intersection of results from the two approaches and that the power to detect cojumps can be improved by taking the union of results from the two approaches.

Lastly, we find some support for Hypothesis 2. There appears to be an association between Federal Funds Target Rate announcements and systematic cojumps.

\section{Acknowledgements}

We thank an anonymous referee for particularly helpful advice. We thank Richard Harris, Kevin Aretz and participants at the 2nd International Conference of the Financial Engineering and Banking Society, the Time-Varying Correlation and Volatility Symposium, Wolverhampton Business School, the Seminar Series of the Accounting and Finance Group, Manchester Business

School and the Seminar Series of the Finance and Accounting Group, Aston Business School for their helpful comments. All remaining errors are ours. 


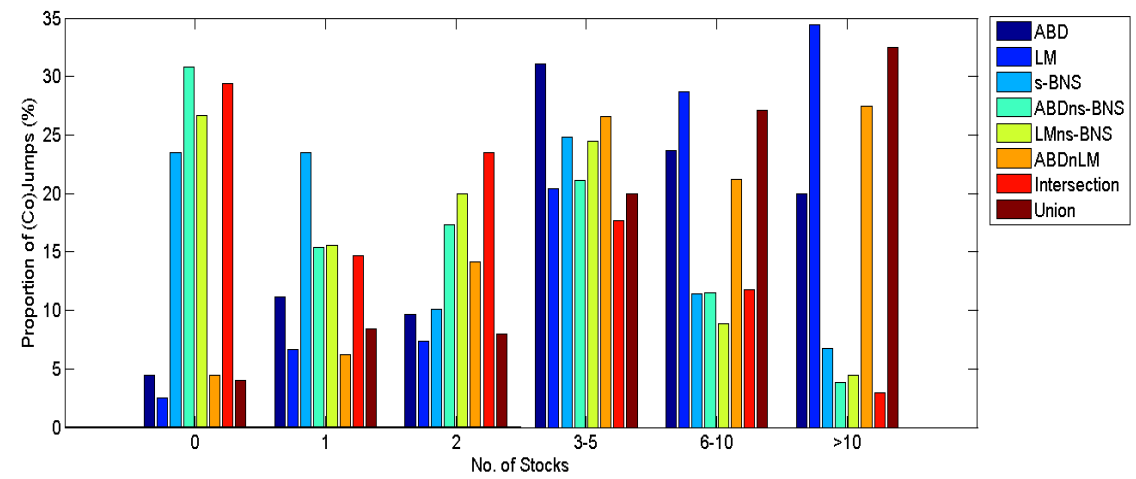

(a)

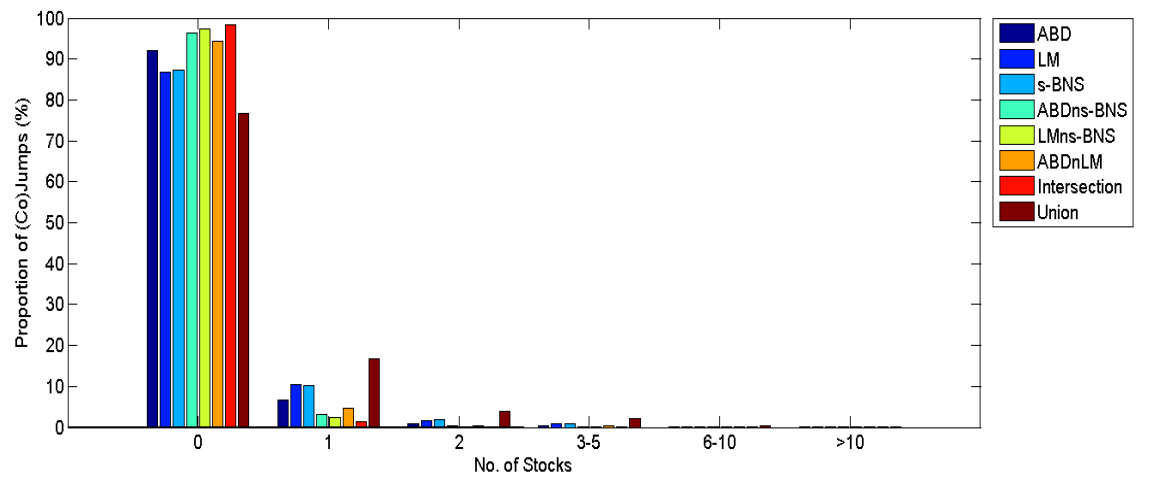

(b)

Figure 1: Systematic and Non-systematic (co)jump distributions for each coexceedance based detection method. Figure 1a presents the proportions of systematic (co)jumps detected to involve different numbers of stocks. Note, the SPY is used as the proxy for the market portfolio and the proportion associated with 0 stocks involved in a systematic (co)jump refers to the proportion of singular SPY jumps, i.e., the proportion of SPY jumps which are not accompanied by cojumps in the individual stocks. Figure $1 \mathrm{~b}$ reports identical information for non-systematic (co)jumps. Note, the proportion associated with 0 stocks refers to the proportion of intraday intervals, not associated with systematic (co)jumps, on which no non-systematic (co)jump occurs. 


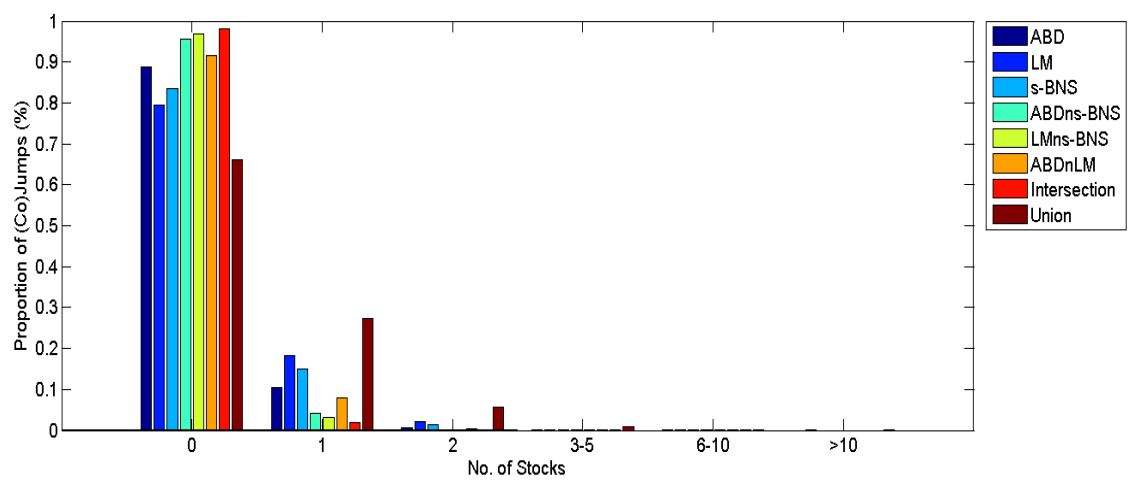

Figure 2: Estimated Poisson-Binomial (co)jump distributions for each coexceedance based detection method if jumps are assumed to be independent. The expected proportions of (co)jumps involving different numbers of stocks, assuming jump arrivals in the stocks are independent, are presented. Note, the proportion associated with 0 stocks refers to the proportion of intraday intervals on which we would expect there to be no (co)jumps. 


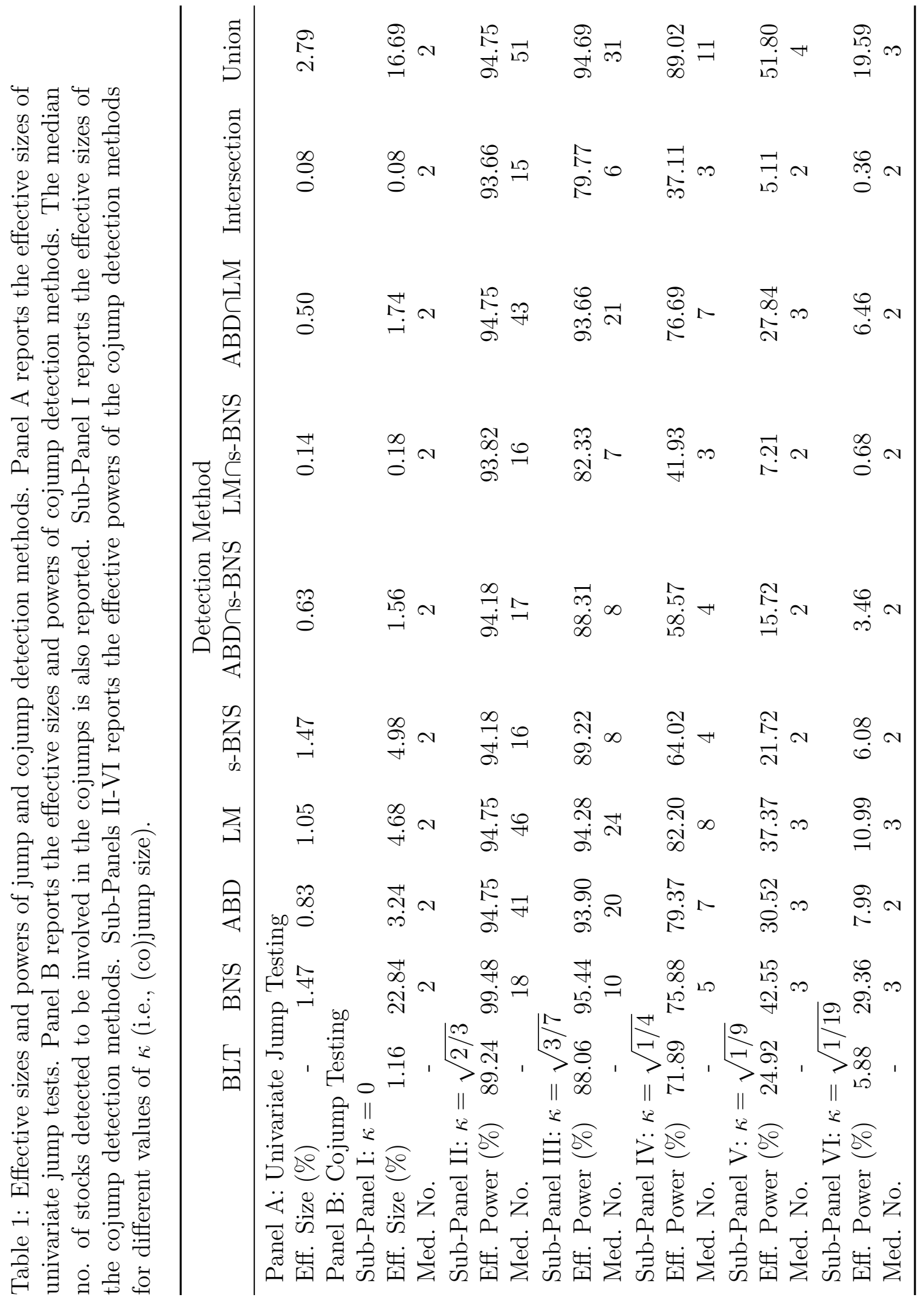


Table 2: Summary of jumps detected in our sample of 61 securities. Columns 1-4 provide the mean number of SPY, total, positive and negative detected jumps. Columns 5-6 provide the mean size of standardised jumps. Column 7 contains the number of stocks for which significant differences ( $p$-value $<0.05$ ) in the number of positive and negative jumps are found according to Chisquared goodness-of-fit tests. Column 8 contains the number of stocks for which significant differences ( $\mathrm{p}$-value $<0.05$ ) in the size of positive and negative standardised jumps are found according to Wilcoxon rank sum tests. Only the mean number of detected jump days is provided for the BNS test.

\begin{tabular}{|c|c|c|c|c|c|c|c|c|}
\hline & \multicolumn{4}{|c|}{ Mean no. Jumps } & \multicolumn{2}{|c|}{ Mean Size } & \multicolumn{2}{|c|}{ Significant Diff. } \\
\hline & SPY & All & Pos. & Neg. & Pos. & Neg. & No. & Size \\
\hline $\mathrm{ABD}$ & 135 & 161.2 & 81.9 & 79.3 & 4.4 & 4.3 & 8 & 5 \\
\hline LM & 314 & 311.0 & 154.6 & 156.4 & 3.4 & 3.4 & 3 & 13 \\
\hline s-BNS & 149 & 244.5 & 123.9 & 120.5 & 3.4 & 3.4 & 3 & 4 \\
\hline $\mathrm{ABD} \cap \mathrm{s}-\mathrm{BNS}$ & 52 & 59.8 & 30.7 & 29.0 & 4.7 & 4.5 & 4 & 2 \\
\hline $\mathrm{LM} \cap \mathrm{s}-\mathrm{BNS}$ & 45 & 43.3 & 23.0 & 20.3 & 4.5 & 4.3 & 2 & 4 \\
\hline $\mathrm{ABD} \cap \mathrm{LM}$ & 113 & 119.1 & 59.9 & 59.2 & 4.4 & 4.3 & 1 & 7 \\
\hline Intersection & 34 & 26.5 & 14.0 & 12.5 & 5.1 & 4.8 & 3 & 7 \\
\hline Union & 450 & 561.4 & 280.7 & 280.6 & 3.4 & 3.4 & 2 & 7 \\
\hline BNS & 129 & 185.7 & & & & & & \\
\hline
\end{tabular}


Table 3: Summary of cojumps detected by each cojump detection method. Panel A contains results for all cojumps. Panel B contains results for systematic cojumps. Panel $\mathrm{C}$ contains results for non-systematic cojumps. Columns 1-2 list the number of detected cojumps and cojump days, across the SPY and 60 firms. Column 3 lists the median number of stocks detected to be involved in the cojumps. Column 4 provides the mean proportion, across all 60 firms, of jumps detected to be involved in cojumps. Two-sample Wilcoxon rank sum tests were conducted to test for a difference in the median number of stocks involved in systematic and non-systematic cojumps. A* (**) next to the median number of stocks involved in a systematic/non-systematic cojump indicates that the median is significantly larger at the $5 \%(1 \%)$ level.

\begin{tabular}{|c|c|c|c|c|}
\hline & No. & Days & Med. No. Stocks & Cojumps (\%) \\
\hline \multicolumn{5}{|c|}{ Panel A: All Cojumps } \\
\hline $\mathrm{ABD}$ & 1182 & 942 & 2 & 45.6 \\
\hline LM & 2587 & 1231 & 2 & 55.9 \\
\hline s-BNS & 2353 & 1467 & 2 & 44.7 \\
\hline $\mathrm{ABD} \cap \mathrm{s}-\mathrm{BNS}$ & 343 & 323 & 2 & 28.4 \\
\hline $\mathrm{LM} \cap \mathrm{s}-\mathrm{BNS}$ & 220 & 204 & 2 & 26.2 \\
\hline $\mathrm{ABD} \cap \mathrm{LM}$ & 826 & 665 & 2 & 47.8 \\
\hline Intersection & 132 & 128 & 2 & 27.6 \\
\hline Union & 5781 & 2130 & 2 & 60.5 \\
\hline BNS & - & 2143 & 5 & 98.7 \\
\hline BLT & 196 & 196 & - & - \\
\hline \multicolumn{5}{|c|}{ Panel B: Systematic Cojumps } \\
\hline $\mathrm{ABD}$ & 129 & 121 & $5^{* *}$ & 10.4 \\
\hline LM & 306 & 245 & $7^{* *}$ & 17.8 \\
\hline s-BNS & 114 & 104 & $3^{*}$ & 3.3 \\
\hline $\mathrm{ABD} \cap \mathrm{s}-\mathrm{BNS}$ & 36 & 35 & 3 & 4.1 \\
\hline $\mathrm{LM} \cap \mathrm{s}-\mathrm{BNS}$ & 33 & 32 & 3 & 4.8 \\
\hline 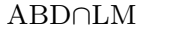 & 108 & 102 & $6^{* *}$ & 13.4 \\
\hline Intersection & 24 & 24 & 2 & 5.6 \\
\hline Union & 432 & 355 & $7^{* *}$ & 13.3 \\
\hline $\mathrm{BNS}$ & - & 126 & $8^{* *}$ & 10.5 \\
\hline \multicolumn{5}{|c|}{ Panel C: Nonsystematic Cojumps } \\
\hline $\mathrm{ABD}$ & 1053 & 855 & 2 & 33.6 \\
\hline LM & 2281 & 1170 & 2 & 36.5 \\
\hline s-BNS & 2239 & 1428 & 2 & 40.1 \\
\hline $\mathrm{ABD} \cap \mathrm{s}-\mathrm{BNS}$ & 307 & 292 & 2 & 23.2 \\
\hline $\mathrm{LM} \cap \mathrm{s}-\mathrm{BNS}$ & 187 & 177 & 2 & 20.2 \\
\hline 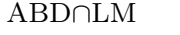 & 718 & 586 & 2 & 32.9 \\
\hline Intersection & 108 & 107 & 2 & 20.9 \\
\hline Union & 5349 & 2088 & 2 & 45.7 \\
\hline BNS & - & 2017 & 5 & 88.1 \\
\hline
\end{tabular}


Table 4: Regression parameter estimates. Panel A presents results from Poisson count regressions. The number of stocks detected to be involved in a cojump, COUNT, determined by each cojump detection method, is regressed against a dummy variable, ISPY, which takes a vlaue of 1 when a jump in the SPY is detected and 0 otherwise. Parameters have been estimated by Method of Moments. Columns 2-3 report parameter estimates with p-values given in parentheses. Columns 4-5 report the number of stocks expected to be involved in a cojump when ISPY takes a value of 0 and a value of 1 . Panel B presents results from linear regressions of jump test statistics. The jump test statistics for the 60 individual stocks are regressed against the jump test statistic for the SPY. The mean value of the estimated intercept $\left(\beta_{0}\right)$ and slope $\left(\beta_{1}\right)$ are reported, with the number of significant parameters (at the $5 \%$ level) given in parentheses. The mean value of the sample correlation between the jump test statistics in the individual stocks and the SPY are also presented, with the number of significant (at the $5 \%$ level) correlations given in parentheses.

\begin{tabular}{|c|c|c|c|c|}
\hline \multicolumn{5}{|c|}{ Panel A: Count Regressions } \\
\hline \multirow[t]{2}{*}{ Detection Method } & \multicolumn{2}{|c|}{ Parameter Estimates } & \multicolumn{2}{|c|}{ Expected Value of COUNT } \\
\hline & $\beta_{0}$ & $\beta_{1}$ & $\mathrm{E}[\mathrm{COUNT} \mid \mathrm{ISPY}=0]$ & $\mathrm{E}[\mathrm{COUNT} \mid \mathrm{ISPY}=1]$ \\
\hline $\mathrm{ABD}$ & $\begin{array}{c}-2.25 \\
(<.0001)\end{array}$ & $\begin{array}{c}4.22 \\
(<.0001)\end{array}$ & 0.11 & 7.14 \\
\hline LM & $\begin{array}{c}-1.68 \\
(<.0001)\end{array}$ & $\begin{array}{c}4.01 \\
(<.0001)\end{array}$ & 0.19 & 10.34 \\
\hline s-BNS & $\begin{array}{c}-1.76 \\
(<.0001)\end{array}$ & $\begin{array}{c}2.92 \\
(<.0001)\end{array}$ & 0.17 & 3.17 \\
\hline $\mathrm{ABD} \cap \mathrm{s}-\mathrm{BNS}$ & $\begin{array}{c}-3.18 \\
(<.0001)\end{array}$ & $\begin{array}{c}4.15 \\
(<.0001)\end{array}$ & 0.04 & 2.63 \\
\hline $\mathrm{LM} \cap \mathrm{s}-\mathrm{BNS}$ & $\begin{array}{c}-3.51 \\
(<.0001)\end{array}$ & $\begin{array}{c}4.48 \\
(<.0001)\end{array}$ & 0.03 & 2.64 \\
\hline $\mathrm{ABD} \cap \mathrm{LM}$ & $\begin{array}{c}-2.59 \\
(<.0001)\end{array}$ & $\begin{array}{c}4.69 \\
(<.0001)\end{array}$ & 0.08 & 8.19 \\
\hline Intersection & $\begin{array}{c}-4.01 \\
(<.0001)\end{array}$ & $\begin{array}{c}4.89 \\
(<.0001)\end{array}$ & 0.02 & 2.41 \\
\hline Union & $\begin{array}{c}-1.03 \\
(<.0001)\end{array}$ & $\begin{array}{c}3.30 \\
(<.0001)\end{array}$ & 0.36 & 9.69 \\
\hline \multicolumn{5}{|c|}{ Panel B: Test Statistic Regressions } \\
\hline \multirow[t]{2}{*}{ Test Statistic } & \multicolumn{2}{|c|}{$\begin{array}{c}\text { Mean } \\
\text { Parameter Estimates }\end{array}$} & \multirow{2}{*}{\multicolumn{2}{|c|}{ Mean Correlation }} \\
\hline & $\beta_{0}$ & $\beta_{1}$ & & \\
\hline $\mathrm{ABD}$ & $\begin{array}{l}0.54 \\
(60)\end{array}$ & $\begin{array}{l}0.34 \\
(60)\end{array}$ & \multicolumn{2}{|c|}{$\begin{array}{l}0.33 \\
(60)\end{array}$} \\
\hline LM & $\begin{array}{c}-0.28 \\
(60)\end{array}$ & $\begin{array}{l}0.36 \\
(60)\end{array}$ & \multicolumn{2}{|c|}{$\begin{array}{l}0.36 \\
(60)\end{array}$} \\
\hline $\mathrm{BNS}$ & $\begin{array}{l}0.47 \\
(60)\end{array}$ & $\begin{array}{l}0.12 \\
(59)\end{array}$ & \multicolumn{2}{|c|}{0.11} \\
\hline
\end{tabular}




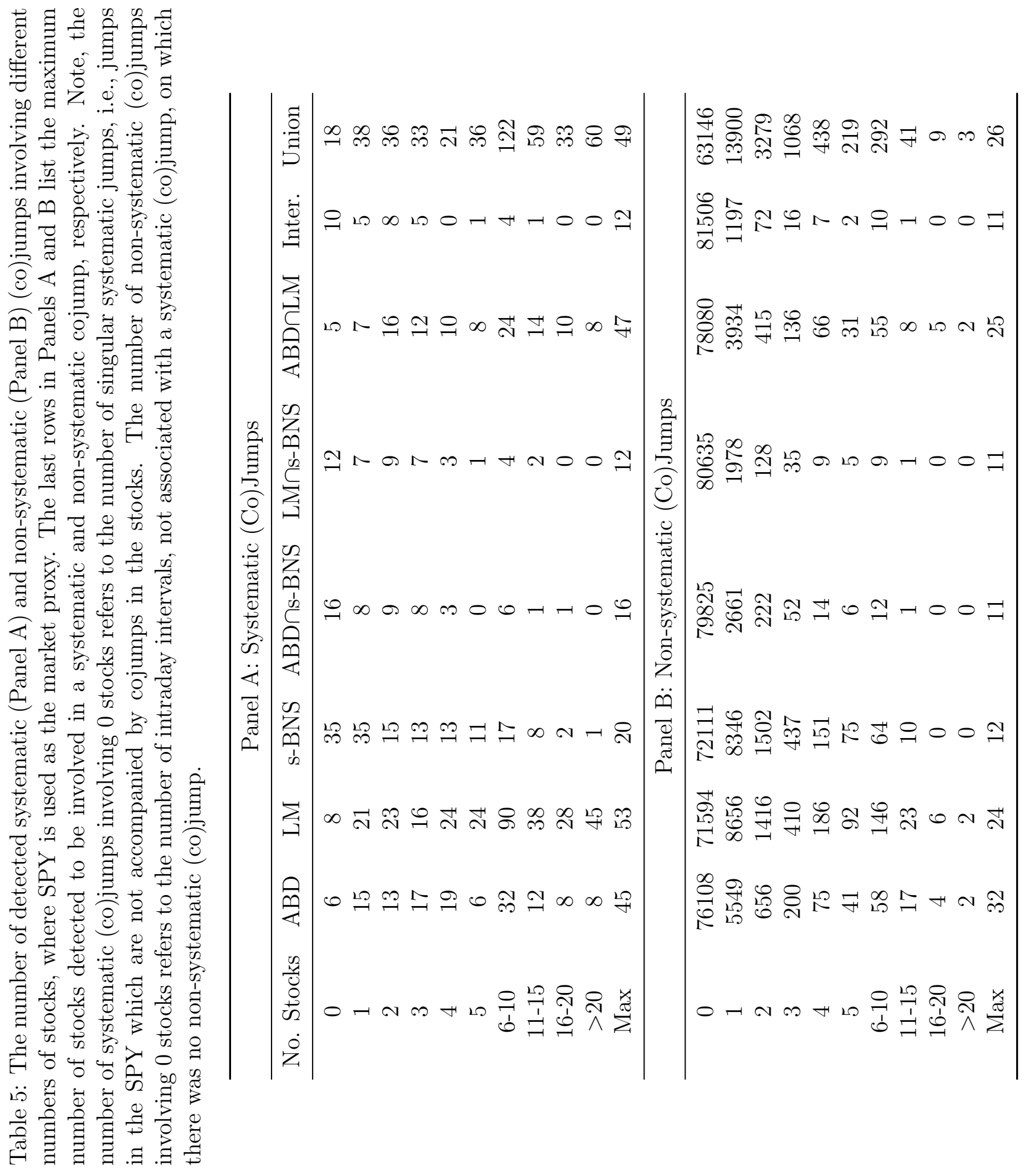




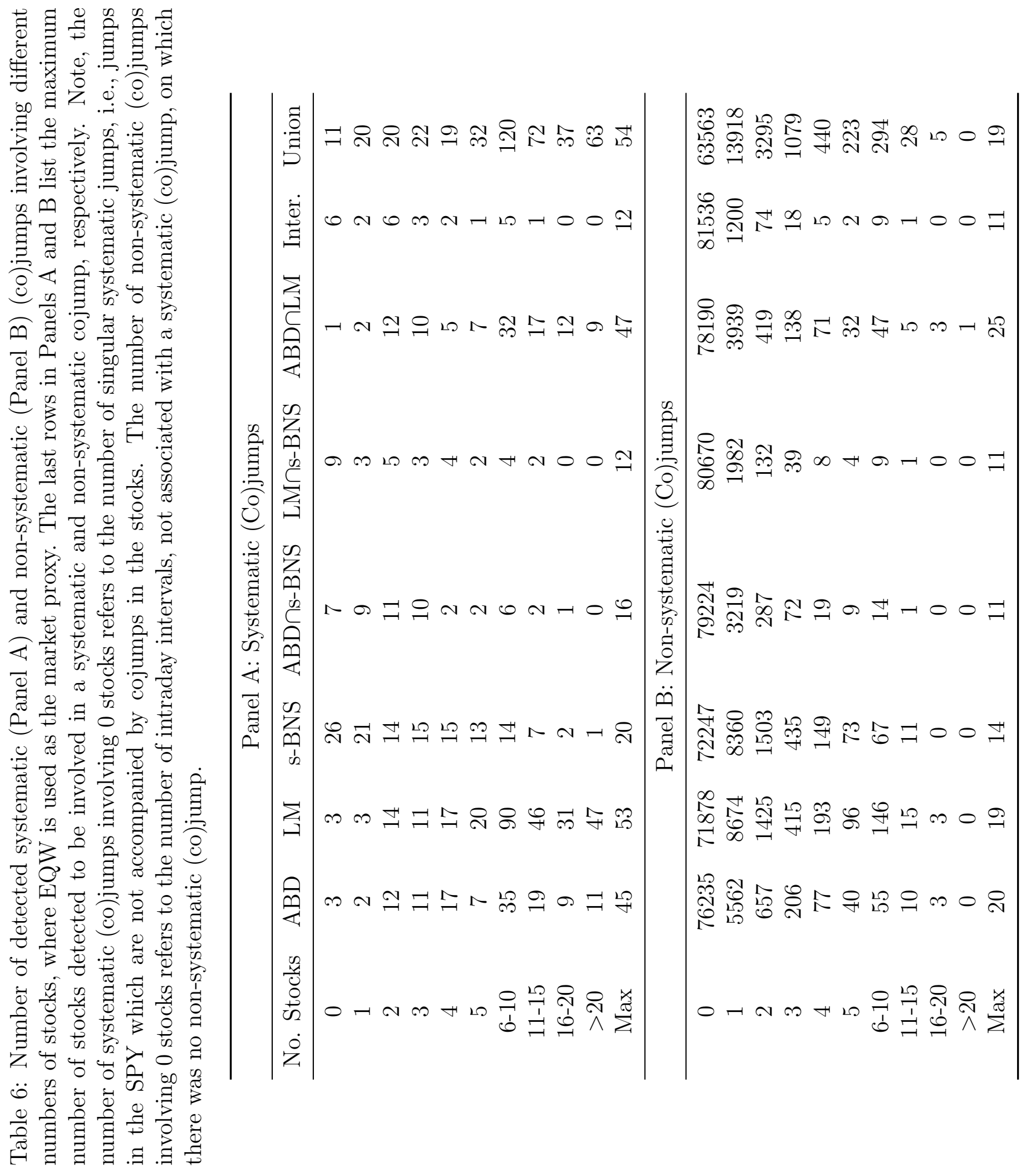




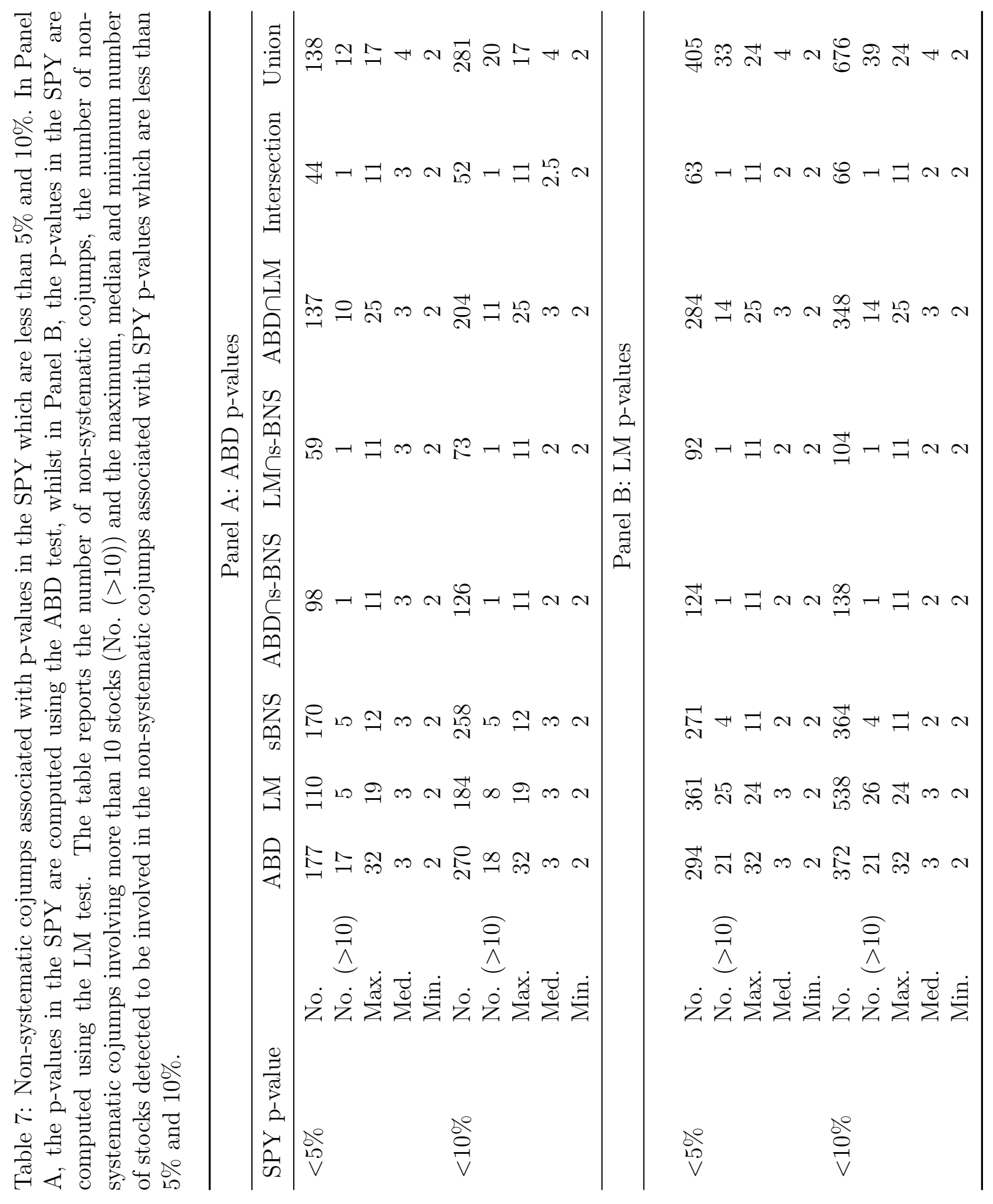


Table 8: Summary of industry cojumps (Panel A) and cojumps with the Fama and French SMB and HML factors (Panel B). In Panels A and B, the number of industry and FF cojumps are reported along with the maximum, median and minimum number of stocks detected to be involved in the industry and FF cojumps. In Panel A column 1, the number of stocks belonging to each industry is also reported. In Panel $\mathrm{B}$, only FF cojumps that occur in isolation of a cojump with the market portfolio are reported, i.e., the association between jumps in the SMB and HML factors and non-systematic cojumps in the stocks is reported. In addition, we also report FF cojumps in which both the SMB and HML factors jump (SMB and HML)

\begin{tabular}{lccccc}
\hline & Stks & $\begin{array}{c}\text { No. } \\
\text { Cojumps }\end{array}$ & $\begin{array}{c}\text { Median } \\
\text { No. Stks }\end{array}$ & $\begin{array}{c}\text { Min } \\
\text { No. Stks }\end{array}$ & $\begin{array}{c}\text { Max } \\
\text { No. Stks }\end{array}$ \\
\hline $\begin{array}{l}\text { Panel A: Industry Cojumps } \\
\text { Industry }\end{array}$ & & & & \\
Consumer Nondurables & 3 & 13 & 2 & 2 & 3 \\
Consumer Durables & 1 & - & - & - & - \\
Manufacturing & 7 & 39 & 2 & 2 & 3 \\
Energy & 3 & 23 & 2 & 2 & 2 \\
Chemicals & 2 & 6 & 2 & 2 & 2 \\
Business equipment & 25 & 880 & 2 & 2 & 8 \\
Telecoms & 1 & - & - & - & - \\
Utilities & 1 & - & - & - & - \\
Retail & 4 & 16 & 2 & 2 & 3 \\
Healthcare & 5 & 51 & 2 & 2 & 3 \\
Finance & 8 & 137 & 2 & 2 & 6 \\
Other & 1 & - & - & - & - \\
Panel B: FF Cojumps & & & & & \\
FF Factor & & & & & \\
SMB & - & 70 & 3 & 2 & 16 \\
HML & - & 33 & 2 & 2 & 23 \\
SMB and HML & - & 1 & 7 & 7 & 7 \\
\hline
\end{tabular}




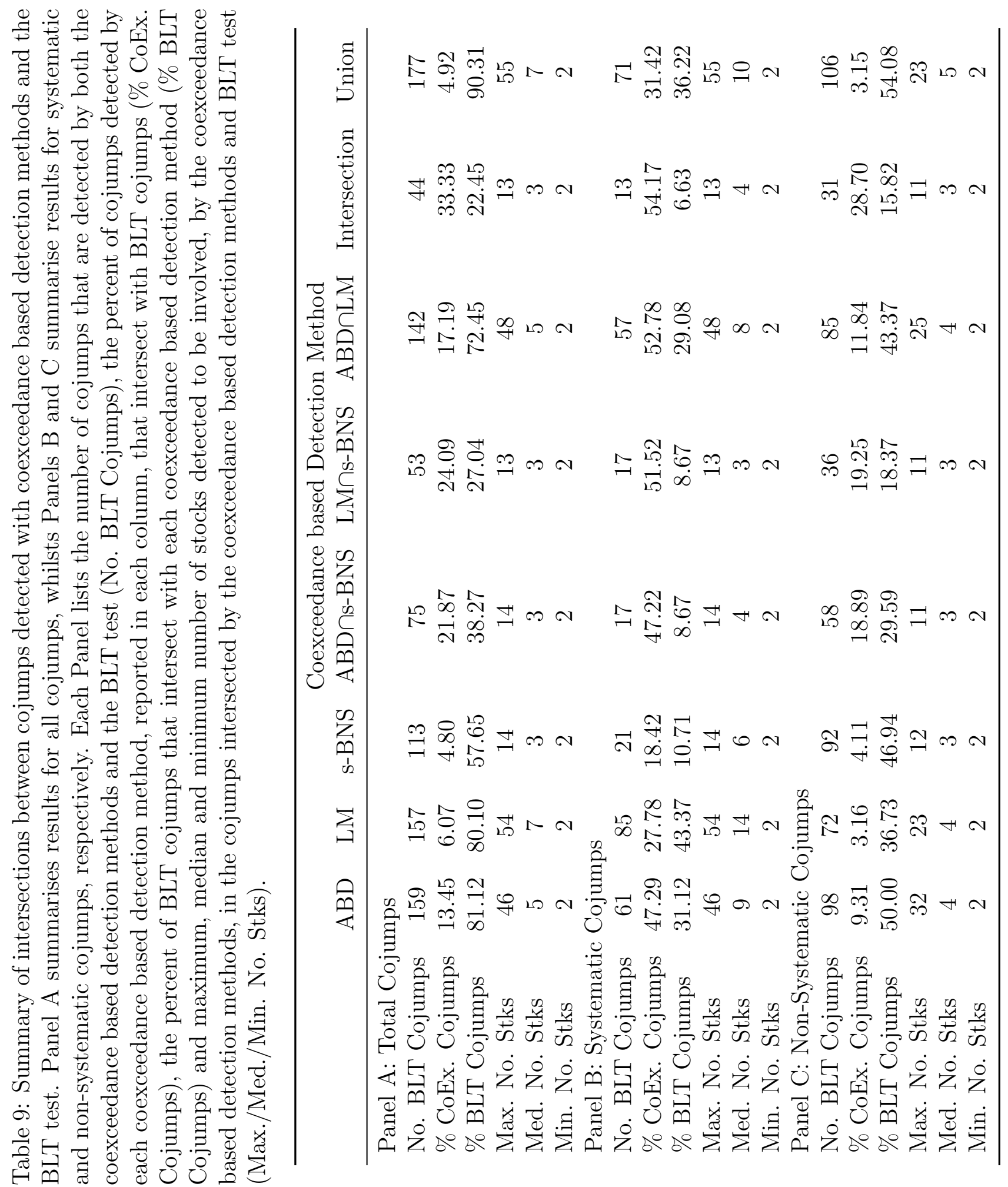


Table 10: Number of systematic cojumps detected if the intersection or union between systematic cojumps detected with coexceedance based detection methods and BLT test is taken.

\begin{tabular}{|c|c|c|c|c|c|c|c|c|}
\hline & \multicolumn{8}{|c|}{ Coexceedance based Detection Method } \\
\hline & $\mathrm{ABD}$ & LM & s-BNS & $\mathrm{ABD} \cap \mathrm{s}-\mathrm{BNS}$ & $\mathrm{LM} \cap \mathrm{s}-\mathrm{BNS}$ & $\mathrm{ABD} \cap \mathrm{LM}$ & Intersection & Union \\
\hline Intersection with BLT & 61 & 85 & 21 & 17 & 17 & 57 & 13 & 104 \\
\hline Union with BLT & 264 & 417 & 289 & 215 & 212 & 247 & 207 & 524 \\
\hline
\end{tabular}




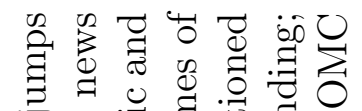

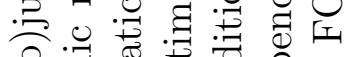

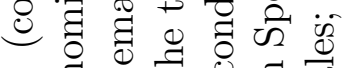

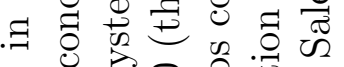

ত

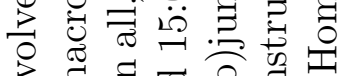

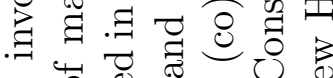

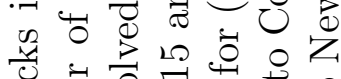

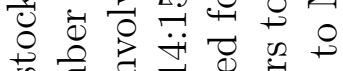

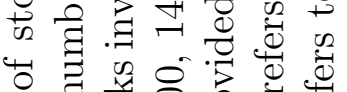

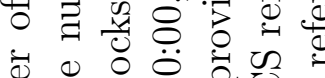

பี

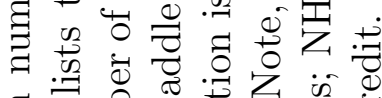

ॠ है है

छ

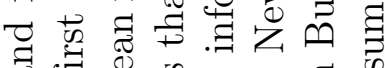

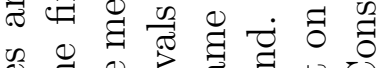
.

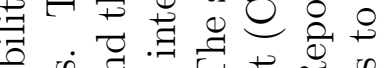

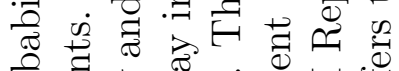

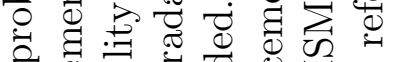

ส

:

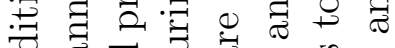

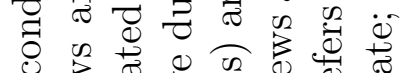

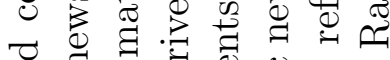

击

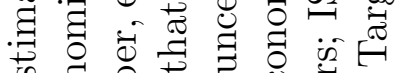

\%

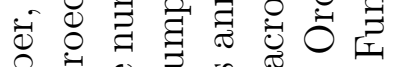

छิ

छ घ्व

() 돈

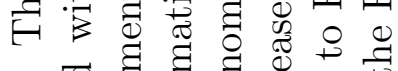

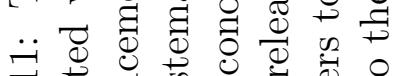

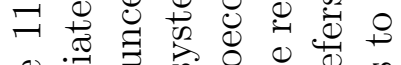

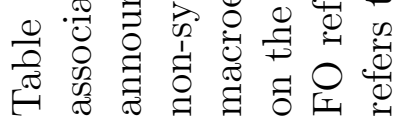

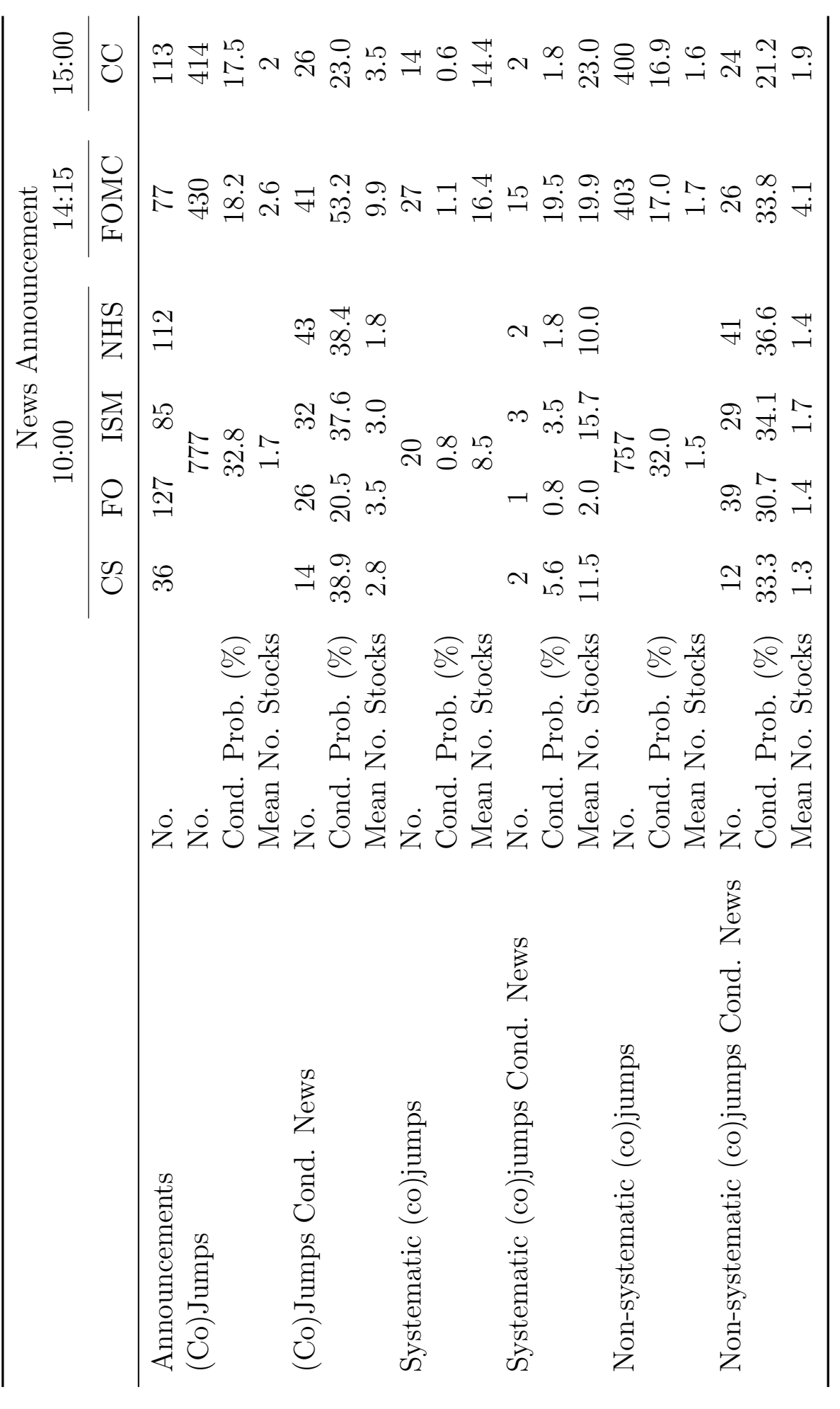


Table 12: Summary of news associated with a sample of systematic cojumps detected by the Union coexceedance detection method. Press articles were obtained from The Associated Press newswire via the Nexis database. The ten dates included in the table are associated with systematic cojumps which involve the ten largest numbers of stocks and do not coincide with macroeconomic news announcements. Columns 1 and 2 provide the date and time (hh:mm) of the systematic cojump. Note the cojump could have occurred up to 11 mins (the length of our intraday intervals) before the time reported in column 2. The number of stocks detected to be involved are listed in column 2. Column 3 summarises news from The Associated Press newswire which is believed to explain the occurence of each systematic cojump. Column 4 indicates whether the jumps in the individual stocks involved in each systematic cojump were positive or negative. Note, all the jumps in the individual stocks involved in the systematic cojumps had identical signs.

\begin{tabular}{|c|c|c|c|c|}
\hline Date & $\begin{array}{c}\text { Time } \\
(\text { hh:mm) }\end{array}$ & $\begin{array}{l}\text { No. Stocks } \\
\text { Involved }\end{array}$ & News & Cojump Direction \\
\hline $03 / 11 / 2010$ & $14: 32$ & 55 & $\begin{array}{l}\text { Fed announces plan to purchase } \$ 600 \text { bn in Trea- } \\
\text { suries. Stocks initially "swung" lower on news. }\end{array}$ & Negative \\
\hline $29 / 09 / 2008$ & $13: 48$ & 55 & $\begin{array}{l}\text { Congress rejects bailout plan. "The blue chip in- } \\
\text { dex [DJIA], dropped by hundreds of points in a } \\
\text { matter of moments..." }\end{array}$ & Negative \\
\hline $06 / 05 / 2010$ & $14: 43$ & 53 & Flash Crash & Negative \\
\hline $26 / 05 / 2010$ & $15: 27$ & 47 & $\begin{array}{l}\text { "A drop in the euro set off a late-day slide in } \\
\text { stocks..." }\end{array}$ & Negative \\
\hline $16 / 03 / 2011$ & $11: 03$ & 43 & $\begin{array}{l}\text { Fear of partial nuclear meltdown at Fukushima. } \\
\text { "Stocks dropped sharply in midmorning trad- } \\
\text { ing..." }\end{array}$ & Negative \\
\hline $06 / 05 / 2010$ & $14: 54$ & 35 & $\begin{array}{l}\text { "Dow Jones industrials fell to a loss of almost } \\
1,000 \text { points in less than half an hour on fears that } \\
\text { Greece's debt problems..." }\end{array}$ & Negative \\
\hline $05 / 02 / 2010$ & $15: 16$ & 33 & $\begin{array}{l}\text { Tentative relationship to Fed announcement that } \\
\text { consumer borrowing decreased less than expected. }\end{array}$ & Positive \\
\hline $12 / 04 / 2005$ & $14: 21$ & 31 & $\begin{array}{l}\text { "Reassuring words from the Federal Reserve trig- } \\
\text { gered late-session stock and bond rallies..." }\end{array}$ & Positive \\
\hline $15 / 01 / 2009$ & $13: 59$ & 30 & $\begin{array}{l}\text { There was "a late burst of buying on Wall Street } \\
\text { on bets the government will again help the finan- } \\
\text { cial industry..." }\end{array}$ & Positive \\
\hline $22 / 02 / 2008$ & $15: 38$ & 29 & $\begin{array}{l}\text { Market "shot" higher in last half-hour of trading } \\
\text { following news that it was likely the bond insurer, } \\
\text { Ambac Financial, would be bailed-out }\end{array}$ & Positive \\
\hline
\end{tabular}




\section{References}

Ait-Sahalia, Y., Chaco-Diaz, J., Hurd, T. R., 2009. Portfolio choice with jumps: A closed-form solution. Annals of Applied Probability 19 (2), 556584.

Andersen, T. G., Bollerslev, T., 1998a. Answering the skeptics: Yes, standard volatility models do provide accurate forecasts. International Economic Review 39 (4), 885-905.

Andersen, T. G., Bollerslev, T., Diebold, F. X., 2009. Parametric and nonparametric volatility measurement. In: Aït-Sahalia, Y., Hansen, L. (Eds.), Handbook of Financial Econometrics. North-Holland, Amsterdam, pp. 67139.

Andersen, T. G., Bollerslev, T., Diebold, F. X., Labys, P., 2000. Great realisations. Risk 18, 105-108.

Andersen, T. G., Bollerslev, T., Diebold, F. X., Labys, P., 2001. The distribution of realized exchange rate volatility. Journal of the American Statistical Association 96 (453), 42-55.

Andersen, T. G., Bollerslev, T., Diebold, F. X., Labys, P., 2003. Modeling and forecasting realized volatility. Econometrica 71, 579-625.

Andersen, T. G., Bollerslev, T., Dobrev, D., 2007. No-arbitrage semimartingale restrictions for continuous-time volatility models subject to leverage effects, jumps and i.i.d. noise: Theory and testable distributional implications. Journal of Econometrics 138 (1), 125-180.

Andersen, T. G., Bollerslev, T., Frederiksen, P. H., Nielson, M. Ø., 2010a. Continuous-time models, realized volatilities, and testable distributional implications for daily stock returns. Journal of Applied Econometrics 25, 233-261.

Andersen, T. G., Dobrev, D., Schaumburg, E., 2010b. Jump robust volatility estimation using nearest neighbour truncation. Working Paper.

Bajgrowicz, P., Scaillet, O., 2011. Jumps in high-frequency data: spurious detections, dynamics, and news. Working Paper.

Bannouh, K., Martens, M., Oomen, R., van Dijk, D., 2010. Realized mixedfrequency factor models for vast dimensional covariance estimation. Working Paper. 
Barndorff-Nielsen, O. E., Hansen, P. R., Lunde, A., Shephard, N., 2009. Realised kernels in practice: Trades and quotes. Econometrics Journal 12 (3), $1-32$.

Barndorff-Nielsen, O. E., Hansen, P. R., Lunde, A., Shephard, N., 2011. Multivariate realised kernels: Consistent positive semi-definite estimators of the covariation of equity prices with noise and non-synchronous trading. Journal of Econometrics 162, 149-169.

Barndorff-Nielsen, O. E., Shephard, N., 2004a. Power and bipower variation with stochastic volatility and jumps. Journal of Financial Econometrics $2(1), 1-37$.

Barndorff-Nielsen, O. E., Shephard, N., 2006. Econometrics of testing for jumps in financial economics using bipower variation. Journal of Financial Econometrics 4 (1), 1-30.

Bollerslev, T., Law, T. H., Tauchen, G., 2008. Risk, jumps and diversification. Journal of Econometrics 144 (1), 234-256.

Bollerslev, T., Todorov, V., Li, S. Z., 2013. Jump tails, extreme dependencies, and the distribution of stock returns. Journal of Econometrics 172, 307-324.

Boudt, K., Croux, C., Laurent, S., 2011. Robust estimation of intraweek periodicity in volatility and jump detection. Journal of Empirical Finance 18 (2), 353-367.

Cameron, C. A., Trivedi, P. K., 1998. Regression analysis of count data. Cambridge University Press, New York.

Chen, S. X., Liu, J. S., 1997. Statistical applications of the Poisson-Binomial and conditional Bernoulli distributions. Statistica Sinica 7, 875-892.

Christensen, K., Oomen, R., Podolskij, M., 2011. Fact or friction: Jumps at ultra high frequency. Working Paper.

Conover, W. J., 1972. A Kolmogorov goodness-of-fit test for discontinuous distributions. Journal of the American Statistical Association 67 (339), 591596.

Corsi, F., Pirino, D., Renò, R., 2010. Threshold bipower variation and the impact of jumps on volatility forecasting. Journal of Econometrics 159, 276288. 
Das, S. R., Uppal, S. R., 2004. Systemic risk and international portfolio choice. Journal of Finance 59 (6), 2809-2834.

Dumitru, A.-M., Urga, G., 2012. Identifying jumps in financial assets: A comparison between nonparametric jump tests. Journal of Business \& Economic Statistics 30 (2), 242-255.

Dungey, M., Hvozdyk, L., 2012. Cojumping: Evidence from the US treasury bond and futures markets. Journal of Banking and Finance 36 (5), 15631575 .

Dungey, M., McKenzie, M., Smith, V., 2009. Empirical evidence on jumps in the term structure of the US treasury market. Journal of Empirical Finance $16(3), 430-445$.

Easley, D., López de Prado, M., O’Hara, M., 2011. The microstructure of the "Flash Crash": Flow toxicity, liquidity crashes, and the probability of informed trading. Journal of Portfolio Management 37 (2), 118-128.

Evans, K. P., 2011. Intraday jumps and U.S. macroeconomic news announcements. Journal of Banking and Finance 35 (10), 2511-2527.

Fama, E. F., French, K. R., 1992. The cross-section of expected stock returns. Journal of Finance 47.

Fama, E. F., French, K. R., 1993. Common risk factors in the returns on stocks and bonds. Journal of Financial Economics 33.

Hansen, P. R., Lunde, A., 2006. Realized variance and market microstructure noise. Journal of Business and Economic Statistics 24 (2), 127-161.

Harris, L., 1986. A transaction data study of weekly and intradaily patterns in stock returns. Journal of Financial Economics 16, 99-117.

Hasbrouck, J., 1999. The dynamics of discrete bid and ask quotes. Journal of Finance 54 (6), 2109-2142.

Huang, X., Tauchen, G., 2005. The relative contribution of jumps to total price variance. Journal of Financial Econometrics 3 (4), 456-499.

Jacod, J., Todorov, T., 2009. Testing for common arrivals of jumps for discretely observed multidimensional processes. Annals of Statistics 37 (4), 1792-1838. 
Jiang, G. J., Lo, I., Verdelhan, A., 2011. Information shocks, liquidity shocks, jumps, and price discovery: Evidence from the U.S. Treasury market. Journal of Financial and Quantitative Analysis 46, 527-511.

Jiang, G. J., Oomen, R. C. A., 2008. A new test for jumps in asset prices. Journal of Econometrics 144 (2), 352-370.

Lahaye, J., Laurent, S., Neely, C. J., 2011. Jumps, cojumps and macro announcements. Journal of Applied Econometrics 26 (6), 893-921.

Lee, S. S., 2012. Jumps and information flow in financial markets. Review of Financial Studies 25 (2), 439-479.

Lee, S. S., Hannig, J., 2010. Detecting jumps from Lévy jump diffusion processes. Journal of Financial Economics 96 (2), 271-290.

Lee, S. S., Mykland, P. A., 2008. Jumps in financial markets: A new nonparametric test and jump dynamics. Review of Financial Studies 21 (6), 2535-2563.

McAleer, M., Medeiros, M. C., 2008. Realized volatility: A review. Econometric Reviews 27 (1-3), 10-45.

Merton, R. C., 1976. Option pricing when underlying stock returns are discontinuous. Journal of Financial Economics 3, 125-144.

Podolskij, M., Ziggel, D., 2010. New tests for jumps in semimartingale models. Statistical Inference for Stochastic Processes 13 (1), 15-41.

Schwert, M., 2011. Hop, skip and jump - what are modern "jump" tests finding in stock returns? Working Paper.

Todorov, V., Bollerslev, T., 2010. Jumps and betas: A new framework for disentangling and estimating systematic risks. Journal of Econometrics 157, 220-235.

Wood, R. A., McInish, T. H., Ord, J. K., 1985. An investigation of transactions data for NYSE stocks. Journal of Finance 40 (3), 723-739. 


\section{Appendix A. Data Cleaning}

To clean the data we used a procedure similar to Barndorff-Nielsen et al. (2009). We purged all transactions for which the correction indicator (CORR) was not zero and the sale condition indicator (COND) was not empty or equal to either ' $E$ ' or ' $F$ ' in the TAQ database. Where an identical time stamp was associated with multiple transactions, we used the median price. We removed prices that were more than 50 mean absolute deviations from the median centered on a window of 50 observations.

\section{Appendix B. Data Selection}

One objective of this study was to obtain a large number of stocks suitable for the application of the jump and cojump tests. However, we also need to be able to accurately estimate BV which, in turn, can only be achieved when the market for the stocks is liquid. That is to say, there must be a sufficient number of transactions observed throughout the trading day for intraday returns to be computed and on which accurate estimates of BV are reliant. To obtain a large sample of liquid stocks, we focused on the constituents of the S\&P 500. Specifically, we selected 100 constituent stocks with the highest trading volumes in January 2002, where, although not directly proportional to the number of trades, trading volume was used as an indicator of liquidity. From this sample stocks were removed as a consequence of merger activity, delisting or missing data, leaving the 60 stocks used in our sample. 